\title{
Adaptive Representations for Improving Evolvability, Parameter Control, and Parallelization of Gene Expression Programming
}

\author{
Nigel P. A. Browne and Marcus V. dos Santos \\ Department of Computer Science, Ryerson University, ON, Canada M5B 2K3 \\ Correspondence should be addressed to Nigel P. A. Browne, nbrowne@acm.org \\ Received 15 September 2009; Revised 6 December 2009; Accepted 11 February 2010 \\ Academic Editor: Oliver Kramer
}

Copyright ( $) 2010$ N. P. A. Browne and M. V. dos Santos. This is an open access article distributed under the Creative Commons Attribution License, which permits unrestricted use, distribution, and reproduction in any medium, provided the original work is properly cited.

Gene Expression Programming (GEP) is a genetic algorithm that evolves linear chromosomes encoding nonlinear (tree-like) structures. In the original GEP algorithm, the genome size is problem specific and is determined through trial and error. In this work, a method for adaptive control of the genome size is presented. The approach introduces mutation, transposition, and recombination operators that enable a population of heterogeneously structured chromosomes, something the original GEP algorithm does not support. This permits crossbreeding between normally incompatible individuals, speciation within a population, increases the evolvability of the representations, and enhances parallel GEP. To test our approach, an assortment of problems were used, including symbolic regression, classification, and parameter optimization. Our experimental results show that our approach provides a solution for the problem of self-adaptive control of the genome size of GEP's representation.

\section{Introduction}

Evolutionary computation (EC) is a machine learning technique that uses processes often inspired by biological mechanisms to obtain a solution to a given problem. Applying an EC algorithm to a problem begins by defining how potential solutions are represented, which is known as the problem representation. A problem representation is defined by the type of input data (the Terminal Set) used to generate a solution, the desired number, and types of outputs and the operations (the Function Set) used to transform the inputs into the output values. An important step in applying an EC methodology to a particular problem is the specification of parameters that define the problem representation and control the algorithm. Finding appropriate parameter values that yield satisfactory results usually requires carefully developed heuristics or expert knowledge. In EC algorithms, the concept of a population of candidate solutions, or individuals, is used to represent a pool of possible solutions to a particular problem. The encodings, or genomes, used to represent a solution vary depending on the EC methodology. It can be as simple as binary code, or as complex as a full fledged programming language. The Gene
Expression Programming (GEP) algorithm [1], developed by Candida Ferreira, is an EC algorithm which uses separate encodings for the genotype and phenotype.

This work introduces novel enhancements to the Gene Expression Programming (GEP) algorithm that enable flexible genome representations, endow self-adaptive characteristics, increase the diversity within a population, and enhances the parallelization of the algorithm. The following issues are particularly relevant to the work presented here.

(1) Evolvability. The structure of the problem representation does not vary during a run, as it is restricted to the initial values for the head domain length and number of genes. This constrains the algorithm to narrow bands of exploration and reduces its ability to produce meaningful change or a paradigm shift within a population.

(2) Crossbreeding and Speciation. In GEP, genetic operations and transformation are restricted to identically structured genomes, preventing different species, or disparately structured genomes, from evolving and competing within a population. 
(3) Distributed Evolution. Parallelization is restricted by the inability for disparate populations to interact, slowing the exploration of the search space.

(4) Parameter Tuning and Self-Adaptation. The GEP algorithm lacks a self-adaptation mechanism and thus requires additional time and resources to systematically evaluate different control parameter sets and subjecting the algorithm to operator biases.

To address the evolvability of the problem representation, we developed two new operators to permit the structure of the GEP genome to be changed during a run. We call these new operators the Adaptive Chromosome Size (ACS) Mutation operator and the Head Insertion Sequence (HIS) Transposition operator.

The problems of speciation and genome interactions between disparately structured individuals were solved by replacing the canonical GEP recombination operators with modified versions that permit dissimilarly structured individuals to interact.

From the beginning of our explorations we wanted to improve the performance of the GEP algorithm when distributed. We quickly realized that transferring individuals between separate GEP populations was severely limited by the inability for structurally different individuals to recombine. This issue was eliminated by the introduction of our modified recombination operators.

Finally, to enable parameter tuning in the GEP algorithm, we designed our HIS and ACS mutation operators to eliminate the two critical parameters of the GEP algorithm: the head size and the number of genes. Additionally, the HIS and ACS mutation operators were designed to permit the algorithm to self-adaptively tune the optimal chromosome structure.

Our proposed methodology was empirically evaluated using an assortment of problem classes and complexity levels. Symbolic regressions evaluated were kinematics problems, a series of polynomial regressions, and the "Sunspot Problem". The classification problem tested was the LiveDescribe dataset from the The Center for Learning Technology at Ryerson University. Finally, the effectiveness of the proposed methodology for optimizing parameters was evaluated using the De Jong test functions [2].

The effectiveness of the proposed changes were evaluated by comparing the performance of the enhanced GEP algorithm against the original GEP algorithm. Additionally, the symbolic regression results were compared to the adaptive distributed GEP algorithm developed by Park et al. [3]. The results obtained using an application developed during the course of this work, known as Syrah, and the results were validated using the K-Fold method with 10 folds.

The specific contributions of this work are as follows:

(1) development of the Head Insertion Sequence (HIS) operator to self-adaptively tune the head size parameter in the GEP algorithm and to enable the structure of the individual to evolve during a run,

(2) creation of the Adaptive Chromosome Size (ACS) Mutation operator that self-adaptively tunes the number of genes of an individual in a GEP population, Therefore allows the genome structure to evolve.

(3) addition of new recombination operators to the GEP algorithm to enable structurally dissimilar genomes to interact, therefore enabling individuals to be transferred between separate GEP populations without any genomic structural constraints. This feature is particularly important to parallel GEP systems, as it permits unrestricted migration.

Following this introduction, we present the background material related to this work in Section 2, our methodology in Section 3, the results and discussions of our experiments in Section 4, and finally, in Section 5, the conclusion and potential future work.

\section{Background}

In this section we present the relevant existing research that pertains to the key issues addressed by this work, including: the canonical Gene Expression Programming algorithm, the evolvability of the problem representation, genome crossbreeding and speciation, distributed evolution, parameter control and, self-adaptation.

2.1. Canonical GEP Algorithm. The Gene Expression Programming (GEP) algorithm was first published by Ferreira in 2001 [1]. Like other EC methodologies, GEP derives its inspiration from biological processes and has been successfully applied to a variety of problems [4-9].

A significant difference in GEP is the separation of the phenotype and genotype. Many existing methodologies, such as Genetic Programming [10] and Genetic Algorithms [11], use a single representation for both the genotype and the phenotype. By separating the representation, the GEP algorithm is able to benefit from the speed of operating on a linear genotype and the flexibility offered by the tree-based phenotype. It also permits the physical representation to affect the genetic code of the individual, as is found in nature.

In the GEP algorithm, each individual or candidate program is referred to as a chromosome. Every chromosome in the population represents a syntactically correct program, because of the underlying nature of the chromosome's encoding and representation.

2.1.1. Chromosome Encoding. In GEP the genome or chromosome consists of a linear, symbolic string of one or more genes, with each gene coding for an expression tree (ET). A gene has two well-defined, adjacent regions called head, containing symbols that code for internal or leaf nodes of the encoded ET, and tail, containing terminal symbols (the leaf nodes) of the encoded ET. In canonic GEP, both the number of genes and the head size of a gene are input parameters for the algorithm. The tail size $t$ is a function of the head size $h$, and is determined as follows:

$$
t=h\left(n_{\max }-1\right)+1,
$$




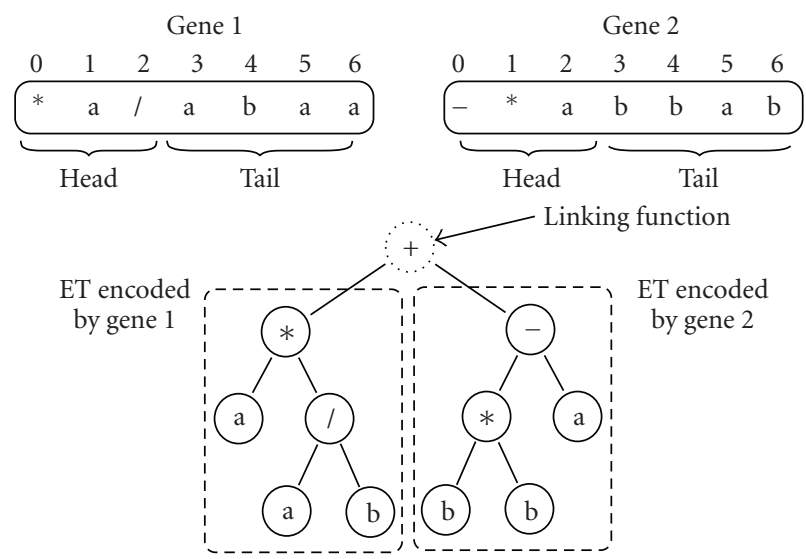

FIgURE 1: Chromosome with two genes: head size 3, tail size 4.

where $n_{\max }$ denotes the maximum arity found in the function set (Like in genetic programming, the function set is also a parameter to the GEP algorithm.).

In the case of multigenic chromosomes, all ETs are connected by their root node using a linking function. In the GEP system presented in this work we used the addition operator as the linking function. To illustrate, Figure 1 shows an example of a chromosome and the respective tree it encodes.

2.2. Evolvability. Evolvability refers to the ability of a genome to change over time and to occasionally produce offspring that are more effective at a particular problem (and thus perform an effective search) [12, 13]. For evolutionary computation, this becomes significant for representations, such as GEP, that separate the phenotype from the genotype. In the case of this work, we focus on the evolvability of the structure of the genotype (the encoding of the genome). This is particularly important in the case of GEP, since the genome structure of the canonical algorithm is fixed throughout a run and controlled by two problem-specific parameters.

Lopes and Weinert [14] proposed an enhanced GEP algorithm called EGIPSYS that varied the length of the head domain on a genome-level basis. The individuals, however, were composed of a fixed number of equal-length genes. This contrasts with the approach presented here, where each individual may have any number of genes and each gene may have a unique head length. Additionally, EGIPSYS neither implemented the one-point recombination operator nor introduced operators to vary a chromosome's length. It also restricted the operation of the gene recombination operator to like-sized individuals. All of these issues are resolved in the method presented here.

In an attempt to improve the evolvability of the individuals in GEP, Yue et al. [15] proposed a crossover strategy called Valid Crossover Strategy which would crossover all individuals in a population and create the subsequent population from the n-best valid chromosomes. This approach seemed to help the evolution of the solution, but not the evolution of the structure itself.
Several different strategies for improving the GEP algorithm were presented by Tang et al. in [16]. A feature of interest that they developed was an adaptive mutation mechanism, which was essentially a fitness proportional mutation rate. On an individual basis, the mutation rate applied to a chromosome was inversely proportional to its fitness. Thus, highly fit individuals would have a lower mutation rate applied to them, reducing the number of potentially disruptive changes to chromosome. Conversely, poorly fit individuals were more likely to have significant mutation performed on their chromosomes. The implementation of the Adaptive Chromosome Sizing Mutation Operator introduced in this work uses the idea of a fitness proportional mutation rate to preferentially mutate the number of genes in poorly fit individuals.

In this work we introduce new operators to improve the evolvability of GEP genomes. The new operators are the HIS transposition and ACS mutation operators, which allow the structure of a GEP genotype to change over time. The evolution of the genotype occurs in parallel, but fundamentally linked, to the exploration of the search space for a particular problem. The two evolutionary processes are interconnected because changes in the genotype can permit the algorithm to explore regions of the search space that may be inaccessible to other genome structures.

2.3. Crossbreeding and Speciation. The concept of crossbreeding and speciation embraced in this work is that of interactions between disparately structured, but fundamentally compatible, genomes. The idea of crossbreeding specifically refers to the ability for any individual, regardless of structure (or species), to reproduce and create viable offspring. The ability to crossbreed any individual permits a more genetically diverse population and enabled unrestricted exploration of the search space by the algorithm.

Speciation, on the other hand, can have several different interpretations. In particular, it can refer to the ability for "subpopulations" to exist within a single main population for the purpose of "niching" [17]. Speciation and niching has been used to promote diversity within a population, prevent (or limit) convergence, and address multimodal problems where different areas of the solution space require different individuals [13]. Two methods for using speciation, or niching, are Crowding [2] and Fitness Sharing [18].

The EGIPSYS algorithm [14] permitted different-sized chromosomes within a population, which other systems, such as canonical GEP, AdaGep [19], and PGEP-O [3], do not support. However, unlike our proposed methodology, all individuals in an EGIPSYS population were required to have the same number of genes. This contrasts with our proposed methodology, which supports (and, in fact, encourages) populations consisting of individuals that have both differing head domain lengths and gene counts.

Park et al. introduced a parallel system, PGEP-O [3], which attempted to dynamically tune specific parameters of the GEP algorithm. In the work, the individuals were constrained by the genome restrictions of canonical GEP; that is, only identically structured individuals were able 
to interact and exist within a single population or island. This methodology was limited because the transfer of individuals between islands, or migration, could only occur between islands with identical gene counts and head domain sizes. The methodology presented in this work eliminates these constraints by creating operators that do not restrict the interaction of genomes with fundamentally different structures.

The contributions presented in this work enable crossbreeding between disparately structured individuals in a GEP population, a feature unavailable in canonical GEP. This enables evolution of different species within a population, and while specifically implementing niching is beyond the scope of this work, it could be examined in the future.

2.4. Distributed Evolution. The intrinsic parallel nature of EC can often be further exploited by distributing a given EC algorithm. Parallelization techniques can generally be classified by their granularity, defined as either fine grained or coarse-grained models. Fine-grained techniques commonly have low computational requirements, but higher communication needs, and are well suited for multiprocessor systems. Coarse-grained models, on the other hand, tend to be computational intensive but have lower communication requirements and are better suited to discrete computational nodes. The Island Model is a coarse-grained technique that was introduced in [20] and has been shown to be fault tolerant [21]. The distributed system implemented to validate our methodology uses the Island Model.

The exchange of genetic material between islands, or demes, is referred to as migration. The structure of the connections between islands, or the topology, is bounded by the cases of isolated islands (no migration) and fullyconnected (migration to all other demes) [22]. Additionally, dynamic topologies have been suggested [23]. In addition to the topology, the rate of migration, number of migrants, and the migration policy control the flow of individuals between islands [24]. One aspect of a migration policy is whether the migration occurs synchronously (migrations occur in specific intervals with specific partners) or asynchronously (migrations occurred whenever a deme has a migrant to exchange) [25]. Interested readers are directed to [23, 24, 2630] for more detailed information regarding migration.

The PGEP-O system [3] is another example of a parallel GEP algorithm which used two island groups. The first island group was a standard Island Model implementation, in which a single population of individuals was evolved on each island. The second island group used the first group's island as their "individuals" in an attempt to use a GA to optimize the parameter settings of the island populations. Since the two island groups were needed, PGEP-O could only operate in a distribute mode. Additionally, since Park et al. did not address the interaction of differing genome structures, migration between the islands could only occur between likestructured populations. This limited the algorithm's ability to explore the search space.

Lin et al. proposed a fine-grained parallel GEP system [31] which exploited niching to improve the performance of the GEP algorithm. Based on their reported algorithm and data, they used a shared pool of like-structured individuals and empirically determined the GEP algorithm's parameter values.

A multiobjective parallel GEP system, PGEP-AP [32], also used the Island Model with migration. In addition to the standard migration mechanism PGEP-AP used a separate elitist population to store the best individuals from the various subpopulations.

The PED-GEP algorithm introduced in [33] used a measure of diversity to guide evolution among parallel clients; however, the details of their parallelization lacked further specifics.

Du et al., in [34], demonstrated a parallel GEP implementation that used Estimation of Distribution to improve the performance of the GEP algorithm. This system used asynchronous migration with a fully connected Island Model; that is, each island (population) could potentially interact with any other island.

Our approach to the distribution of the GEP algorithm was to use a fullyconnected coarse-grained model with random migration and to remove the restrictions placed on the migration mechanism by canonical GEP's inability to support dissimilarly structured chromosomes in a single population. By permitting unrestrained migration, populations in a parallel setting are now able to freely exchange candidate solutions to enhance the solution quality and diversity.

2.5. Parameter Control and Self-Adaptation. Most Evolutionary Computation algorithms require a set of control parameters, which influence the process evolution to be configured based on the particular problem being explored. The process of setting these parameters often requires complex heuristics, "rules of thumb", or specific knowledge from a domain expert. Thus, it is desirable to automatically tune the parameter values prior to executing the algorithm or to self-adaptively tune the parameters during the run.

In problem solving and optimization, the impossibility theory of "No Free Lunch" [35] has been postulated and roughly states that without a priori knowledge of a problem (to tailor the methodology to it) no single problem-solving method is inherently better for all problem classes [36]. This has implications for any evolutionary algorithm and parameter control method, especially those with attempt to optimize the parameters prior to executing an evolutionary run and then use static values throughout the run [37]. Additionally, it has been shown [38] that optimal parameter values can vary throughout a single run. This implies that, while it may be impossible to determine optimal values for all problems and situations, it should be possible to evolve values that are "good enough". Additionally, it implies that methodologies that are able to optimize their parameter values dynamically have an inherent advantage over those that do not.

The PGEP-O system presented in [3] approached the issue of parameter control as a separate optimization problem that ran in parallel to the main evolutionary algorithm. 
This system used a parallel GEP implementation, using the Island Model, to evolve solutions to the target problem and a genetic algorithm (GA) running on a separate client to optimize the two GEP parameters. The head size and gene count parameters were optimized by using trial values on each GEP island and then reporting back to the GA parameter optimizer. This approach, while successful, suffered from several issues that are remedied by our proposed methodology. The PGEP-O algorithm required additional resources, since the parameter optimization was a separate calculation. Additionally, the GA optimizer had to wait for an entire run to complete before it was able to execute a new generation, which is problematic for long-running evolutions.

The DM-GEP algorithm [33] introduced a dynamic mutation rate operator in an attempt guide evolution. $D M$ GEP divided the execution of a run into three stages, the initial stage; the metaphase stage, and the anaphase stage. Each stage was then assigned a specific mutation rate and the mutation rate used in each generation was progressively scaled, by a fixed amount, from one value to the next. In this manner, the number of generations executed in a run was directly related to the mutation rates. This approach did not, strictly speaking, tune the mutation parameter and was not self-adaptive, but did dynamically alter the rate and showed improvement over the standard GEP implementation.

Bautu et al. introduced in [19] an algorithm, called AdaGEP, for automatically controlling the number of genes of a GEP representation. The approach involved adding to the genome a bit array that maps each bit to a gene in the chromosome. The bit in each position of the array indicates if whether gene would be included in the translation to an expression tree during the fitness evaluation. Specific genetic operators were designed to operate on this bit array, thus evolving an optimal mask. The AdaGEP algorithm was limited by the fact that the total number of genes in any chromosome could never change. Thus, there was little benefit to using that method versus using automatically defined functions, or homeotic genes, in GEP's jargon, to evolve the execution order of the genes. Additionally, the size of individuals in the algorithm's population could never change, so that, even if fewer genes were required, the genetic operators would still be performed on the full chromosome.

The work presented in [15] included a method to vary the mutation and crossover rates during a run, based on the Cloud Model [39]. This methodology improved the performance of the GEP algorithm, but was only applied to like-structured genomes.

Eiben et al. stated in their "Parameter Control in Evolutionary Algorithms" survey [37] that determining successful values for algorithm parameters in EC is a "grand challenge" problem.

The approach to parameter control and self-adaptation presented in this work was accomplished using multiple techniques which work together to self-adaptively tune GEP parameters. To tune the head domain length and number of genes, we developed the HIS transposition and ACS mutation operators. In addition to these operators, we created new recombination operators which allowed structurally disparate (and normally incompatible) genomes to be able to crossbreed and create viable offspring, which permits individuals with different head domain length and gene count parameters to compete within a single population.

\section{Methodology}

This section introduces the proposed enhancements to the GEP algorithm to address the issues identified in Section 1, to wit the evolvability of the problem representation, genome speciation and crossbreeding, distributed evolution, and parameter control and self-adaptation in the canonical GEP algorithm. The section will introduce our proposed enhancements, the details of the implementation of the framework used for evaluation, and the experiments used to validate our hypothesis.

3.1. Proposed GEP Algorithm Enhancements. To address the issues of evolvability, crossbreeding, distributed evolution, and parameter control found in canonical GEP, our proposed modifications to GEP include several new operators and also modifications to the existing recombination operators. The new operators introduced in the following section offer solutions to the problems of evolvability and the control of two critical parameters in GEP. The modified recombination operators were developed to permit speciation within a GEP population and to enhance distributed GEP populations.

The original version of the GEP algorithm required that two critical parameters, the length of the head domain and the number of genes in the chromosome, to be set to fixed values prior to the execution of a run. These parameters are generally domain and problem specific, which further exacerbates the problem of finding "good" values (not even particularly optimal ones) for the parameters. By developing new operators which permit genome structure changes, we enabled the head domain length and number of genes to be implicitly tuned during a run. Our algorithm enhancements also permit each gene in a chromosome to have a unique head domain length. This extra feature enables the length of the gene to vary, and thus the length of the function encoded by that gene.

In addition to parameter control, our approach improves the evolvability, or the ability of the structure of the genome to evolve, by removing the fixed-length chromosome restrictions in canonical GEP and allowing the number of genes to vary during a run. Chromosome evolvability was specifically addressed by designing the new operators to increase the capacity of the genome for extracting and exploiting the underlying structure of the fitness function under consideration.

The new operators for parameter control and enhancing evolvability are presented in Sections 3.1.1 and 3.1.2.

3.1.1. Adaptive Chromosome Size Mutation Operator. In Algorithm 1 we present the pseudocode for the Adaptive Chromosome Size (ACS) mutation operator used in our enhanced GEP algorithm. The ACS operator mutates the number of genes in a chromosome, potentially increasing or 


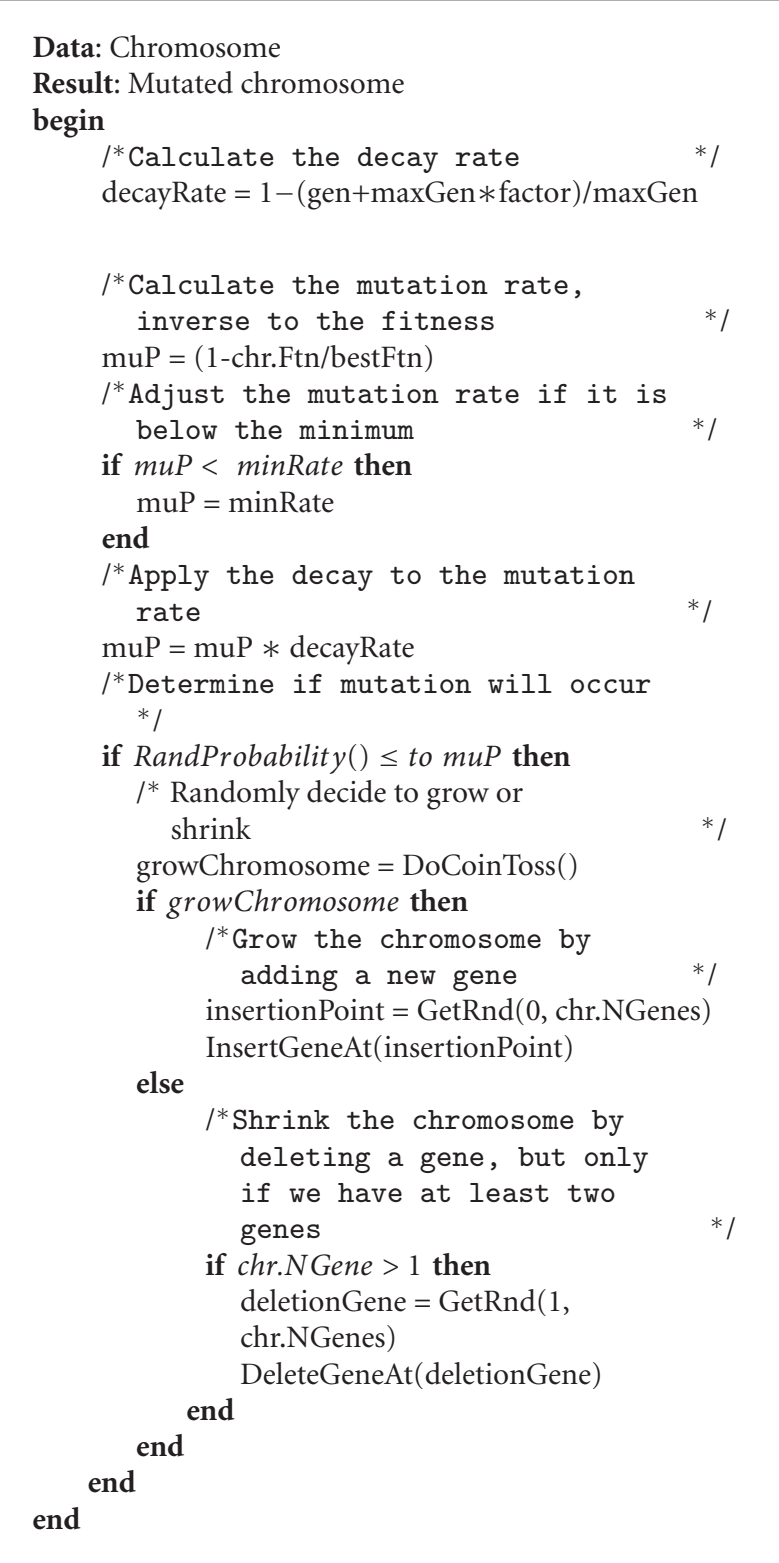

Algorithm 1: ACS mutation operator pseudocode.

decreasing the total number of genes when it is applied. The ACS operator is applied to the entire population during each generation.

The AcsGeneMutation $(\cdots)$ method takes a chromosome (chr) as a parameter and mutates it according to the following procedure. Initially, it calculates the decayRate, which is used to decrease the operator's application as the run progresses. In the decayRate calculation the factor is a user-defined value that scales the decayRate and is set to 0.2 for all experiments. This scales the decayRate to zero for the final 20 percent of the run.

Next, the algorithm calculates the probability of mutation $m u P$. The probability of mutation is inversely proportional to the individuals fitness when compared to the best fitness in the current generation. If the $m u P$ is less than the user-defined minimum mutation rate, minRate, then $m u P$ is set equal to minRate. The mutation rate, $m u P$, is then scaled by decayRate to arrive at the final $m u P$ value. The operator then generates a random probability using RandProbability () and compares it to $m u P$ to determine whether the AcsGeneMutation will be applied to the chromosome. Next, the operator performs a coin toss using DoCoinToss () to determine whether a gene should be added or removed. When a gene is added, the operator selects an insertion point, insertionPoint, at a random position in the sequence of genes of the chromosome.

It then calls the worker method, InsertGeneAt $(\cdots)$, to insert a randomly created gene at the insertion point. When a gene is removed, the operator first verifies that there is more than one gene (chr.NGenes) in the chromosome. It then randomly selects a gene in the chromosome using the GetRnd $(\cdots)$ method and calls the DeleteGeneAt $(\cdots)$ method to remove the gene from the chromosome.

The mutation operator always uses a step size equal to one. Thus, it modifies a single gene in the chromosome during each application of the operator. Alternative step sizes were not investigated, but will be examined in future work.

3.1.2. HIS Transposition Operator. To dynamically tune the size of a gene, we introduced a new transposition operator called head insertion sequence transposition, HIS transposition, for short. The transposable elements (also called transposons) in this case are fragments of the genome, located in the head of a gene, that can be activated and jump to (possibly) another gene head in the chromosome. Two features make this operator different from the canonic transposition operators used in GEP, to wit that

(i) the transposable element is necessarily located in the head of a gene,

(ii) during transposition the transposon is cut from the place of origin (instead of copied, like in canonic transposition in GEP), thus shortening the length of the respective gene, and then inserted in the place of destination located necessarily in the head of (possibly) another gene, thus elongating the gene length at the target site.

Specifically, the HIS transposition operator works as follows. Initially the operator randomly chooses the chromosome, the start and end sites of the transposon, and the target site. As mentioned above, these start and end sites are located in the head of a gene. Moreover, transposons contain at most three elements. Next, the operator cuts the transposon from the site of origin, making the necessary arrangements to maintain the structural integrity of the gene. That is, if the transposon locates in the middle of the head of a gene, then the left and right remaining segments of the head are concatenated, thus forming the new gene head. Next, the operator inserts the transposon at the target site, thus elongating the head of the gene. Notice that the gene heads at the place of origin and at the target site have now changed; the latter is now longer by, say, $k$ elements, and the former is $k$ elements shorter. Finally, using (1), the operator adjusts 
the respective new tail sizes of those genes. If the tail requires extra material, it is taken from the remaining genetic material in the source gene's tail.

\subsubsection{Recombination Operators for Nonuniform Chromo-} somes. The notion of species is not present in canonic GEP, as all chromosomes have the same structure; that is, all individuals in a population have the same gene head size, same gene tail size, and the same number of genes. The possibility of different species within a single GEP population is highly desirable feature for the parallelization of the algorithm, particularly when using a migration mechanism in a distributed setting. By modifying the existing GEP recombination operators to handle genomes with different structures, our enhanced GEP algorithm now supports crossbreeding and speciation within both a single population and distributed islands.

To support different-sized chromosomes created by ACS mutation and HIS transposition operations, we created modified versions for the one-point and two-point recombination operators used in GEP. These operators also facilitate integrating individuals with differing genome structures (i.e., a differing number of genes and head domain lengths) into a target population during migration, when distributed. Recombination via these operators works as follows: initially the first positions for the head and tail sections of the two-parent chromosomes are paired (see Figure 2). Then the crossover point (or points, in the case of two-point recombination) is randomly chosen from the overlapping sections of the chromosome. Then the crossover point locates either in the head of a gene or in the tail. If it falls in the head, then the genetic material is exchanged (the strands swapped) at the crossover point (see Figure 2(a)). For this case, there is no need to adjust the structure (tail size) of the gene containing the crossover point. If it locates in the tail of a gene, then we use the following process to exchange the genetic material of the genes where the crossover point is located. First we exchange the genetic material at the point of crossover. Then, we verify that the tail sizes of the resulting genes comply with the respective resulting head sizes. If the tail size of a recombined gene is $s$ elements shorter than the allowed size, then we append to it $s$ elements from the tail of the other parent gene, thus making the final tail size of the recombined gene compliant with its head size (notice the strand added to $O_{1}$ in Figure 2(b)). On the other hand, if the tail size of the recombined gene is symbols longer than the allowed size, then we cut its $s$ last symbols out (notice the strand removed from $\mathrm{O}_{2}$ in Figure 2(b)).

The rest of genetic material is exchanged as in normal crossover, with a caveat: for the case of GEP-RNC (GEP with real number constants [7]), if the crossover point locates in the tail of a gene, the genetic material in the domain of constants (Dc) is exchanged as normal and the lengths of the Dc domains are adjusted. If the crossover point falls in the Dc domain, then recombination proceeds via the same procedure used for the tails, as illustrated in Figure 2(b). The arrays containing the gene's real number constants are exchanged in their entirety [40].

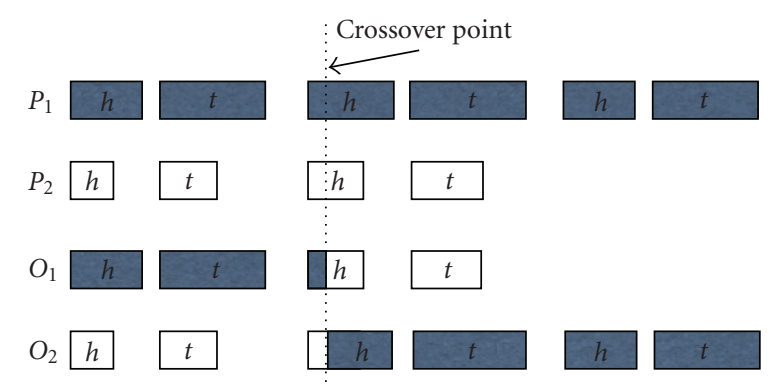

(a)

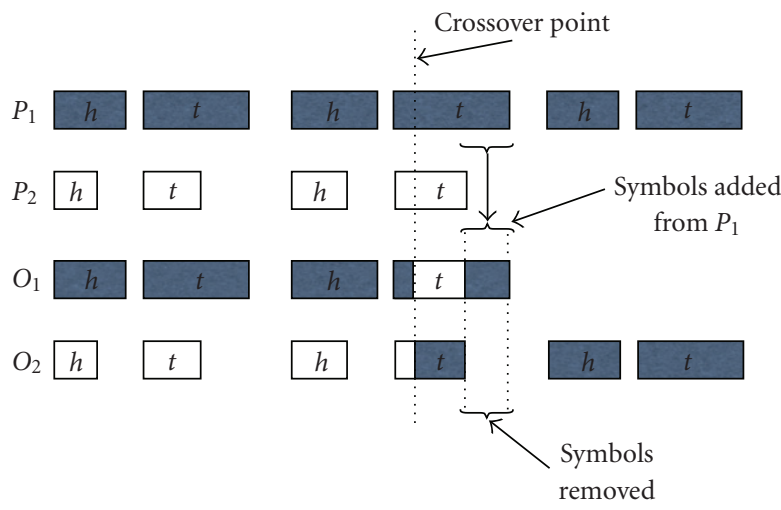

(b)

Figure 2: One-point recombination of two chromosomes, $P_{1}$ and $P_{2}$, containing 3 and 2 genes, respectively; $h$ and $t$ denote the head and tail portions of each gene, respectively. In Figure 2(a) the crossover point locates in the head of a gene. In Figure 2(b) the crossover point locates in the tail of a gene.

Analogous to GEP, our recombination operators also produce two children from the parents, with one child having the same length as one of the parents, and the other child having the same length as that of the other parent.

3.2. Syrah Implementation. In this study, a parallel capable GEP system called Syrah, which dynamically tunes the number of genes and gene size, was developed. To test this system, a suite of nontrivial symbolic regressions was used and the quality of the models was benchmarked against models obtained via a canonic GEP system and competing methodologies.

Syrah's system requirements differ from GEP-RNC (GEP with real number constants [7]) in regards to the genetic operators it uses, which are detailed in Sections 3.1.1, 3.1.2, and 3.1.3.

In Syrah's implementation, tournament selection with elitism was used. Many GEP implementations use Roulette Wheel selection, but as long as elitism is used, various selection methods will produce equally good results [7].

When the Syrah system is operating in parallel, it uses a coarse-grained model (or Island Model [20]) to distribute the populations. Syrah uses the proposed genetic operators to permit disparate genome structures to be integrated into a given population during a migration event. 
3.2.1. Development and Runtime Environments. All components of the research system Syrah were written in C\# using the Microsoft.Net Framework version 3.5 and developed using Microsoft Visual Studio 2008. Data storage and management was accomplished using Microsoft SQL Server 2005 running on Microsoft Windows XP Professional. The client computers also used the Microsoft Windows XP Professional operating system.

3.2.2. Parallelization. Different methods and techniques exist for operating an EC algorithm in parallel. Generally parallel techniques can be divided into two categories: fine grained and coarse grained [24]. Fine-grained techniques involve parallelizing the evaluation of the test cases and usually have more intensive communication requirements. Alternatively, coarse-grained techniques distribute populations and have lower communication requirements, but higher computational needs. Our experimental system uses a common coarse-grained technique known as the Island Model [20] to distribute populations to discrete computational nodes. The Island Model implemented in Syrah is a fullyconnected topology that supports random-random migrations, meaning that a migration event can (randomly) involve any node in the system. Details regarding migration can be found in [22-24, 26, 27, 29, 41].

The network communication between nodes was implemented using the HTTP v1.1 protocol over an SSL connection. The server node is designed to listen for client requests on port 443, the standard port used by SSL web servers. Additionally, the communication between the client and the server is always initiated by the client. This combination of techniques was selected so that the communication would be relatively secure and to facilitate communication between the client and server, when the client was located behind a firewall. This was done to circumvent firewall issues in the original network used for testing.

Finally, based on [21], the nodes do not implement any special handling for detecting and preventing network topology faults. When a client is unable to complete a run (i.e., the host was restarted, network failure, etc.), the client is simply to starts a new run when it rejoins the Syrah topology.

3.2.3. Population Initialization. With the use of our recombination operators, the population is able to support individuals with different chromosome sizes. To take advantage of this feature, the population is seeded with randomly sized chromosomes. Both the number of genes and the head domain length of each gene are varied during this phase. The number of genes in each individual is randomly selected between 1 and 10. During the creation of the chromosome, each gene selects a random head domain length between 5 and 15 . These values were empirically determined during initial testing and were found to provide good genetic diversity. Additionally, we selected the random initialization method over a "ramped half-and-half" method [10] as a result of early experimentation.

The elements of the head are selected from a weighted bag. If the function set is smaller than that of the terminals, then the probability of selecting a function is $1 / 2$; otherwise they are equally weighted.

3.3. Experimental Design. An assortment of problems, of varying types and difficulty, were selected to evaluate the performance of our approach. The problems were selected from three areas to which Evolutionary Computation is commonly applied:

(i) symbolic regression, or the automatic synthesis of functions,

(ii) classification, or generating boolean results (or labels) from a set of input values,

(iii) parameter optimization, or the automatic discovery of parameter values which produce a maximum and/or minimum for a given function.

3.3.1. Validation of Results. Each experiment was performed using K-Fold validation with 10 folds and 30 runs per fold. Each experiment consisted of two sets: a baseline set and an adaptive set. The baseline runs were executed using the standard GEP-RNC algorithm implemented as a part of the Syrah system with parameter control disabled. The adaptive runs were then executed in the same manner, but using the methodologies outlined previously.

Each experiment was executed using the Syrah framework's parallel mode, which uses the Island Model to distribute the populations to separate computational nodes. The experiments used 32 islands that were executed on 16 dual-core Intel computers, running the Windows XP Professional operating system. The Syrah system supports migration between the islands But to facilitate the statistical analysis of the results, these experiments were run without this feature.

The baseline experiments were performed repeatedly using the values presented in Table 1. During the adaptive evolution runs, the number of genes and the size of the head domain were tuned using our new operators. The details of the initial chromosome lengths can be found in Section 3.2.3.

3.3.2. Symbolic Regression Experiments. The first three problems selected were the same problems used by Park et al. in [3]. These were selected so that the performance of this methodology could be compared to an existing (parallel) GEP-based self-adaptive approach. The fourth experiment was a regression of a sawtooth wave, while the fifth experiment was a more difficult time series analysis problem. The baseline experiments all produced poor results for gene counts of 1 through 3 , which required $900(3 \times 10$ folds $\times 30$ runs per fold) runs to determine.

Experiment 1. The first problem evaluated was a kinematics symbolic regression that modeled the movement of a vertically fired object. The kinematic equation for the position of the object at time $t$ is defined by the following equation:

$$
S(t)=S_{0}+V_{0} t+\frac{a t^{2}}{2} .
$$


TABLE 1: Common experiment run parameters.

\begin{tabular}{lcc}
\hline Parameter & Problem & Classification \\
\hline Selection method & Symbolic Regression \& Parameter Optimization & Elitist Tournament \\
Number of generations & Elitist Tournament & 175 \\
Population size & 100 & 75 \\
Initial head size & 100 & $5-15$ \\
Initial number of genes & $5-15$ & $1-10$ \\
One point recombination rate & $1-10$ & 0.5 \\
Two point recombination rate & 0.5 & 0.1 \\
Gene recombination rate & 0.1 & 0.1 \\
Mutation rate & 0.1 & 0.07 \\
Minimum ACS mutation rate & 0.07 & 0.05 \\
IS transposition rate & 0.05 & 0.1 \\
RIS transposition rate & 0.1 & 0.1 \\
HIS transposition rate & 0.1 & 0.1 \\
Gene transposition rate & 0.1 & 0.1 \\
Function set & 0.1 & $F_{2}$ \\
Linking function & $F_{1}$ & + \\
K-Fold validation & + & 10 folds \\
Evolutionary Clients (Syrah) & 10 folds & 31 \\
\hline
\end{tabular}

$F_{1}=\{+,-, *, /\}, F_{2}=\{+,-, *, /$, sqrt, exp, sin, cos, tan, floor, ceiling, OR, AND, $<,>, \leq, \geq,==, !=\}$.

If we use an initial velocity, $V_{0}=25 \mathrm{~m} / \mathrm{s}$, and an initial position of $S_{0}=0$ and assume that the acceleration is equal to earth's gravity, $a=-9.8 \mathrm{~m} / \mathrm{s}^{2}$, then we can simplify the equation as

$$
S(t)=25 t+\frac{-9.8 t^{2}}{2}=25 t-4.9 t^{2}
$$

For this experiment, fifty data points were sampled from the interval $t=0.1$ to $t=5$ and used as the test cases.

Experiment 2. Our second experiment extended the first, using two independent variables instead of one. Modifying (2) with the same assumptions as in Experiment 1, but with an independent initial velocity, gives:

$$
S(t)=v t-4.9 t^{2} .
$$

The test cases for this experiment were generated using $V_{0}$ values of 20,25, and 30 . The values of $t$ were the same as in the first experiment.

Experiment 3. The third symbolic regression experiment used a fourth-order polynomial that was used in [3] and similar to the ones used in $[1,7]$ :

$$
y=-2.5 x^{4}+4.6 x^{3}+3 x^{2}+2 x+1
$$

The algorithm attempted to evolve the function from 10 equally spaced samples taken from values of the Polynomial (5), in the interval $x=[1,10]$.
Experiment 4. The fourth experiment was a regression of a sawtooth wave, which has been used as a benchmark in other works [42]. The function is defined by

$$
F(x)=\sum_{i=0}^{n}\left(\frac{1}{i} \sin (i \quad x)\right): \quad n=1, \ldots, 9 .
$$

The dataset consisted of 250 equally spaced data points in the range $x=[-8,8]$. This range was selected instead of the 40 points in $[-1,1]$ used in [42] after discovering that the algorithm required a more challenging set of inputs.

Experiment 5 (Wolfer Sunspot Time Series Prediction). The final experiment attempted to create a predictive model using 100 observations from the well-known Wolfer Sunspot Series [43]. The data were formatted for time series analysis, using a delay time of 1 and an embedding dimension of 10 . This dataset has also been used to evaluate other GEP systems, including those in [7] and [14].

3.3.3. Classification Experiment. Classification is a common and important task for evolutionary computation algorithms. The classification experiment performed in this work used a large, real-world classification problem from the The Centre for Learning Technology (CLT) at Ryerson University.

The evaluation of the classification experiments was accomplished using the "Hits with Penalty" method, as described in [7].

The LiveDescribe project [44] is a software application developed by the Center for Learning Technology (CLT) at Ryerson University to added video descriptions (for the deaf) to video content. The project had originally used a manual process to select regions of dialog versus nondialog, so that 
descriptive video captions could be programmatically added to the non-dialog sections. Since the process of selecting the nondialog regions was a manual and user-intensive process, the CLT modified their application using a human-designed classifier system. This system was, on average, $70 \%$ effective.

The dataset consists of six real-value inputs and a single boolean output. Part of what makes this dataset a challenge is its size. The initial dataset consisted of approximately 90,000 records. The input variables are audio metrics and include RMS standard deviation, RMS average, a measure of audio entropy, zero crossing above to below, zero crossing left skew, and a zero crossing low-energy measurement. These inputs were sampled once for every 1 second of audio.

3.3.4. Parameter Optimization Experiments. The five parameter optimization test functions were selected from the the well-known De Jong test functions [2]. These test functions were originally selected by De Jong to test the effectiveness of a given EC algorithm over a broad class of problems. While attempts have been made to improve the test set, it remains the de facto standard for parameter optimization validation. The five functions are presented here in their original form, but were modified (where necessary) to change them all to maximization functions, which allows for simpler evaluation with the GEP algorithm.

De Jong F1: Sphere Model. The first function in the De Jong test set is a three-dimensional parabola that is convex, unimodal, and continuous. The function has a maximum of 78.6 at $\left(x_{1}, x_{2}, x_{3}\right)=( \pm 5.12, \pm 5.12, \pm 5.12)$ :

$$
f(x)=\sum_{i=1}^{3} x_{i}^{2}:-5.12 \leq x \leq 5.12 .
$$

De Jong F2: Rosenbrock's Function. The second function in the De Jong test set was first proposed by Rosenbrock [45] and is commonly referenced in optimization literature. This function is nonconvex, unimodal, and continuous, with a maximum of 3905.93 at $\left(x_{1}, x_{2}\right)=(-2.048,-2.048)$ :

$$
f(x)=100 \times\left(x_{1}^{2}-x_{2}\right)^{2}+\left(1-x_{1}\right)^{2}:-2.048 \leq x \leq 2.048
$$

De Jong F3: Step Function. The third De Jong test function is a five-dimension step function that is discontinuous, nonconvex, unimodal and, piecewise constant. De Jong original selected this function to test the ability for algorithms to handle discontinuities [2]. This function is restricted to $-5.12 \leq x \leq 5.12$ for testing. This function has a known maximum of 25 when the inputs are held at 5.12:

$$
f(x)=\sum_{i=1}^{5} x_{i}:-5.12 \leq x \leq 5.12 .
$$

De Jong F4: Quadratic Function with Noise. The fourth test function in the De Jong collection is a noisy quadratic function that is continuous, unimodal, convex, and haing a
TABLE 2: Summary of symbolic regression experimental results.

\begin{tabular}{lcccc}
\hline Exper. & \multicolumn{2}{c}{ Ours } & \multicolumn{2}{c}{ Comparison } \\
Number & Length & Fitness & Length & Fitness \\
\hline $1^{1}$ & 254 & $99.984 \%$ & 266 & $99.496 \%$ \\
$2^{1}$ & 87 & $99.983 \%$ & 282 & $99.907 \%$ \\
$3^{1}$ & 155 & $99.735 \%$ & 470 & $96.187 \%$ \\
$4^{2}$ & 62 & $99.987 \%$ & 185 & $99.966 \%$ \\
$5^{2}$ & 55 & $99.179 \%$ & 186 & $98.936 \%$ \\
\hline
\end{tabular}

1: Compared to PGEP-O.

2: Compared to canonical distributed GEP.

high dimensionality. The function uses a Gaussian function to add noise. The function was limited to $-1.28 \leq x \leq 1.28$. This experiment used alternative values for the number of generations and the population size of 350 generations and 500 individuals in the population.

The maximum of this function is approximately 1248.2 and occurs when all inputs are equal to \pm 1.28 :

$$
f(x)=\sum_{i=1}^{30} i \times x_{i}^{4}+\operatorname{Gauss}(0,1):-1.28 \leq x \leq 1.28 .
$$

De Jong F5: Shekel's Foxholes. This is a two-dimension function that is continuous, nonquadratic, and non-convex, with 25 local maximums and was originally suggested by Shekel [46]. This version [47] of the function has maximum of approximately 499.002:

$$
\begin{aligned}
f(x, y)= & 500 \\
& -\frac{1}{0.002+\sum_{j=0}^{24} 1 /\left[1+i+(x-a(i))^{6}+(y-b(i))^{6}\right]},
\end{aligned}
$$

where

$$
\begin{gathered}
a(i)=16 \times(i \bmod 5-2), \\
b(i)=16 \times\left(\left\lfloor\frac{i}{5}\right\rfloor-2\right)-65.523 \leq x \leq 65.523 .
\end{gathered}
$$

\section{Results and Discussion}

This section presents the results of the experiments outlined in Section 3 that were used to validate our enhancements to the GEP algorithm that address the issues identified in Section 1 .

4.1. Symbolic Regression Results. Table 2 shows a summary of the experiment results, including the best individual's fitness and chromosome size (Note that the size of a chromosome (i.e., the length of the chromosome string) depends on its number of genes and the head size of each gene). The best fitness is expressed as a percentage of the number of fitness cases solved. The visualized results and performance of the experiments are shown by Figures 3, 2, 4, 5, 6, 7, 8, 9, 10, 11 and 12. 


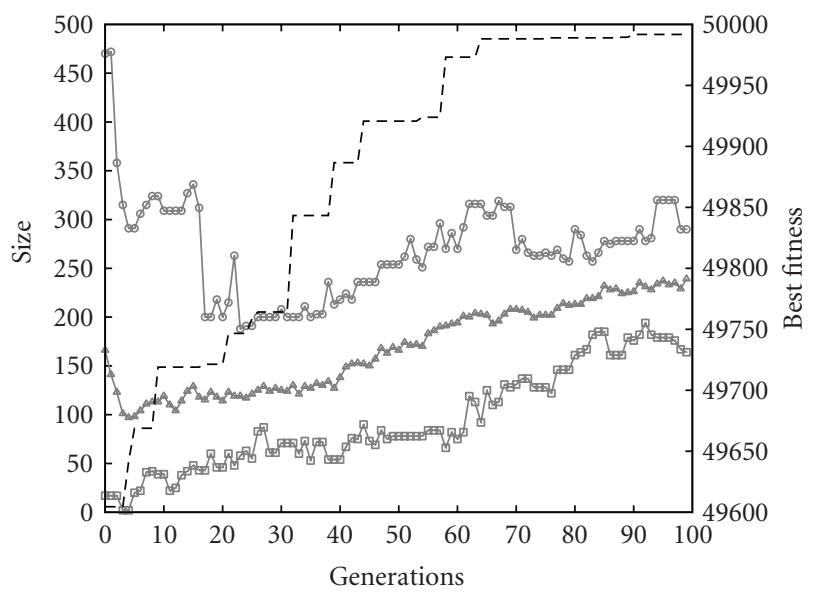

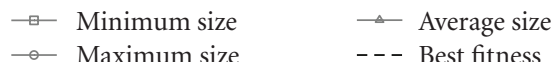

Figure 3: Symbolic regression Experiment 1: chromosome sizes and best fitness.

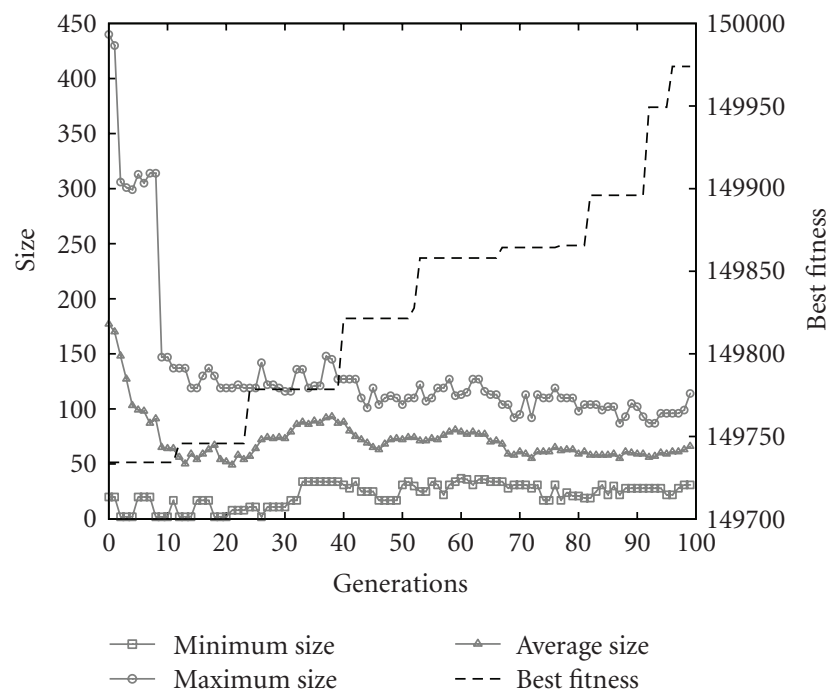

FIGURE 4: Symbolic regression Experiment 2: chromosome sizes and best fitness.

4.1.1. Discussion of Symbolic Regression Experiments. There are two figures for the first four experiments performed. The first figure of each pair shows the minimum, maximum, and average chromosome lengths in the population for each generation with respect to the generation number in the run. The other figures display a surface visualization of the distribution of the chromosome lengths in the population, with respect to the generation number in the run. For the final experiment, the surface plot was omitted because of the rapid convergence to a narrow range of chromosome lengths. Figure 11 compares the evolved model's performance to the target data. Since K-Fold validation was used, every tenth data point in Figure 11 was previously unseen by the model.

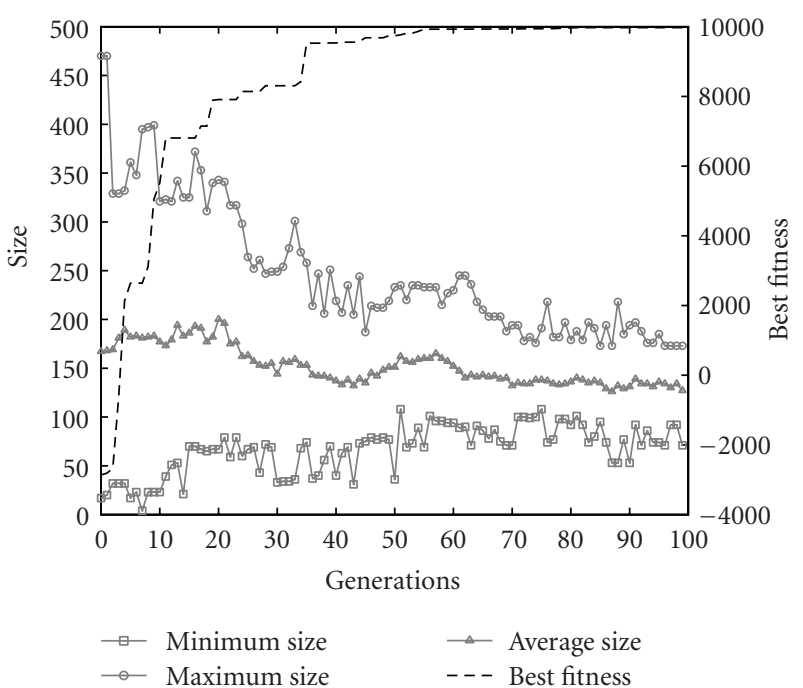

FIGURE 5: Symbolic regression Experiment 3: chromosome sizes.

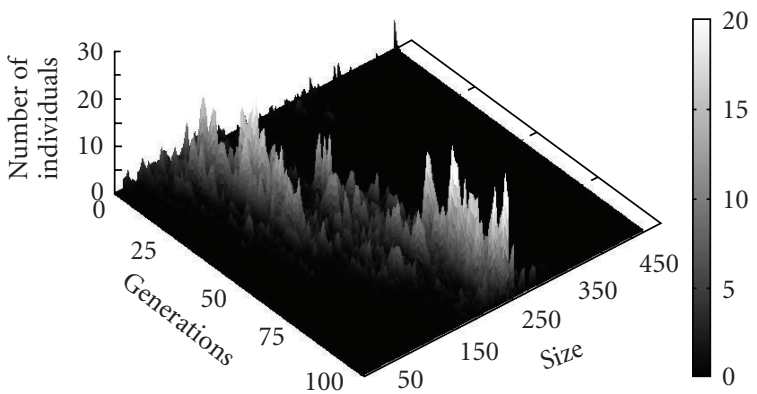

FIGURE 6: Symbolic regression Experiment 1: chromosome size in the population.

The figures show that while the algorithm was optimizing the chromosome length it would initially explore a wide search space, then focus on a band of neighboring chromosome sizes.

A significant result was that the best solutions found using our new operators evolved better individuals with smaller representations than the PGEP-O system presented in [3] and the canonical GEP algorithm. It is interesting to note that the best chromosomes evolved for the two most difficult problems were significantly smaller than those evolved by the PGEP-O. Specifically, during the second and third experiments, the best evolved individuals were approximately $30 \%$ to $33 \%$ of the size of the individuals evolved using the PGEP-O methodology. Similarly, in Experiments 4 and 5, where our methodology was compared to a distributed canonical GEP algorithm (based on Syrah), our methodology produced results $33 \%$ and $30 \%$ the size of the alternative's results.

The results of the experiments, as shown in Table 2, show that our new operators are significantly more efficient and produced better results for symbolic regression problems. This may have been because our populations were evolving smaller solutions and were able to explore the search space more effectively. 


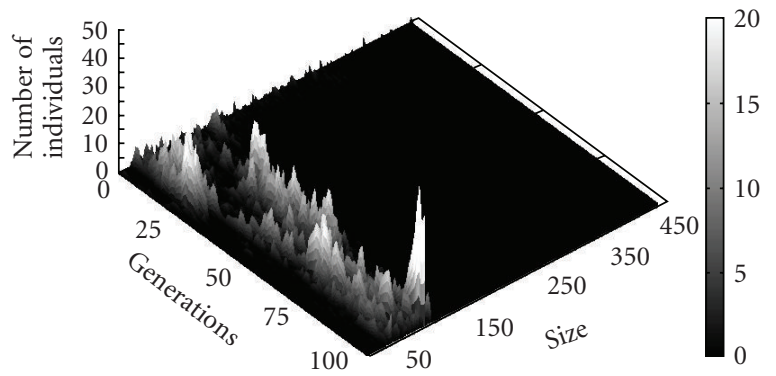

FIgURE 7: Symbolic regression Experiment 2: chromosome size in the population.

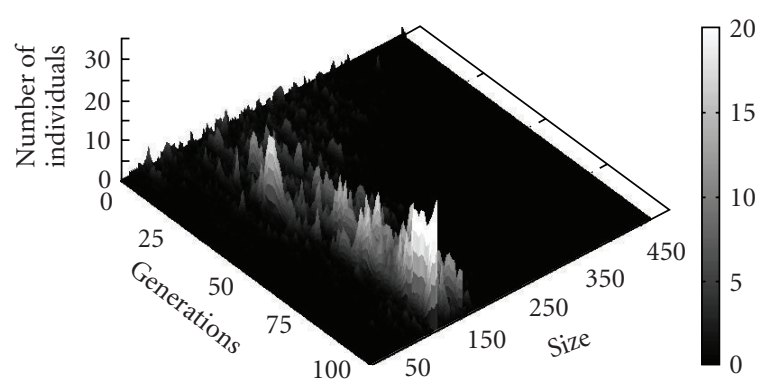

Figure 8: Symbolic regression Experiment 3: chromosome size in the population.

4.2. Classification Results. Table 3 shows the results of the classification experiments. These include the chromosome size and the best fitness found, expressed as a percentage of the number of fitness cases solved. The visualized results and performance of the experiments are shown by Figures 13 and 14.

4.2.1. Discussion of Classification Experiments. As stated in Section 3, the full LiveDescribe dataset consisted of approximately 90,000 entries, each with 6 real number variables and grouped into two classes. One of the challenges of this experiment was the computational resources required to evolve candidate solutions.

The two methods both evolved individuals with similar performance, with both systems evolving a classifier capable of successfully identifying $80 \%-81 \%$ of the fitness cases. This is a substantial improvement over the original, humanwritten classifier (developed by the CLT at Ryerson [44]), which was able to correctly classify approximately $70 \%$ of the fitness cases. After discussions with the CLT lab, it is believed that $85 \%$ may be the practical limit for identifying non-dialog sections of video using the current variable set. The CLT is currently working to modify its data acquisition software to collect additional parameters.

Examining the solutions evolved by our enhanced algorithm and canonical GEP, it is important to note that our methodology evolved a solution $32.1 \%$ the size of the one evolved by the standard algorithm. Since the size of the candidate solution's genome has a direct impact on the evaluation of the fitness cases (and live data, once implemented in the real world), the reduction in representation size may

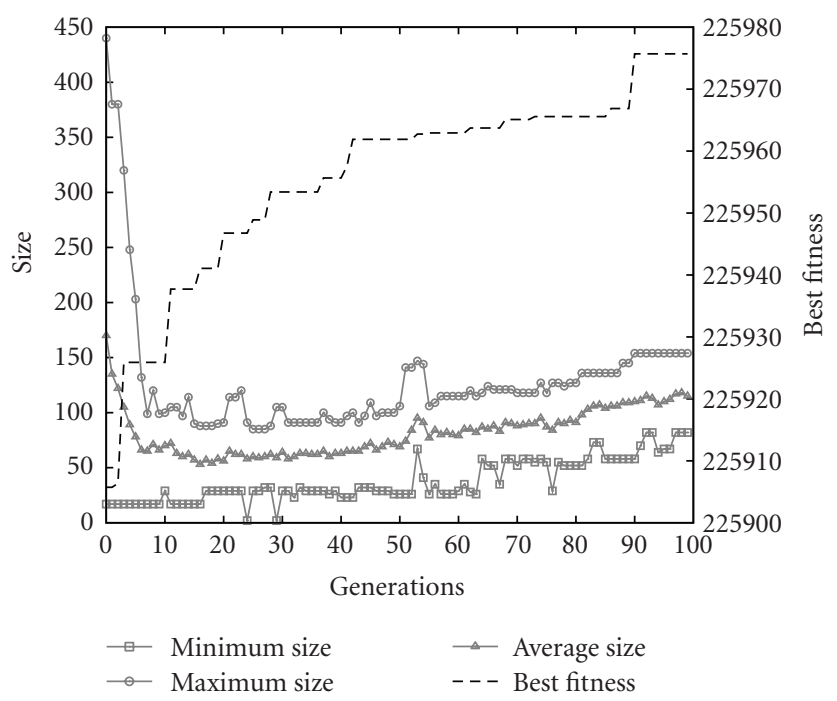

FIGURE 9: Symbolic regression Experiment 4: chromosome sizes and best fitness.

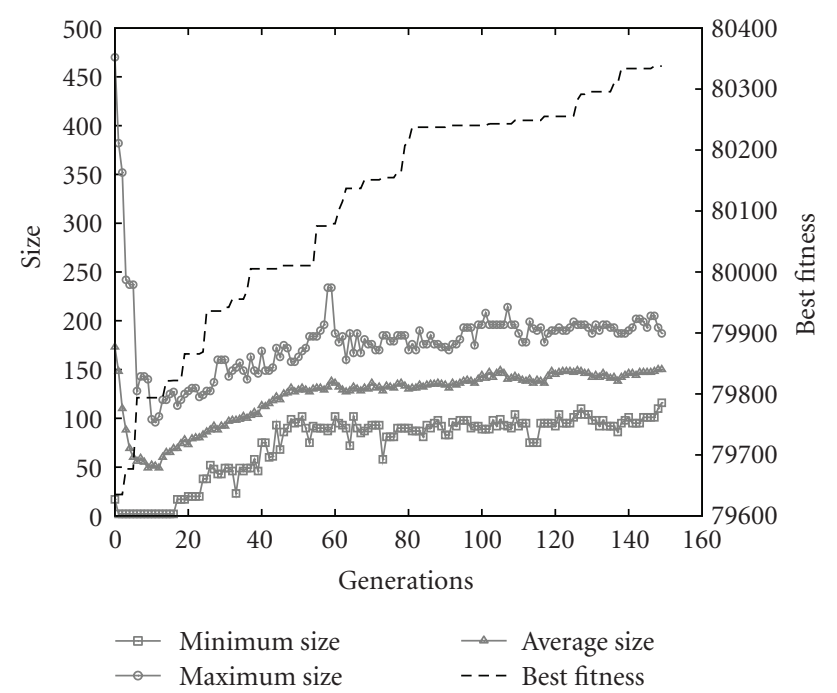

FIgURE 10: Symbolic regression Experiment 5: chromosome sizes and best fitness.

improve the overall performance of the system, even after considering the additional computation requirements of our new operators.

The small number of classes in this experiment may have been possible limitation. With only two possible classes, the evolutionary process may not have been significantly challenged. However, it is felt that the number of test cases may have offset this. In the future, more complex classification problems should be investigated.

What the summary of results do not show is the number of additional epochs (and thus processing time) required to evaluate different values for the head domain length and number of genes for the canonical GEP algorithm that was used for comparison. 
TABLE 3: Summary of classification experimental results.

\begin{tabular}{lcccccrr}
\hline & \multicolumn{3}{c}{ Ours } & \multicolumn{3}{c}{ Canonical GEP } \\
Total Len. & Genes & Avg. Gene Len. & Fitness & Total Len. & Genes & Gene Len. & Fitness \\
\hline 247 & 8 & 30.9 & $81.08 \%$ & 770 & 10 & 77 & $80.31 \%$ \\
\hline
\end{tabular}

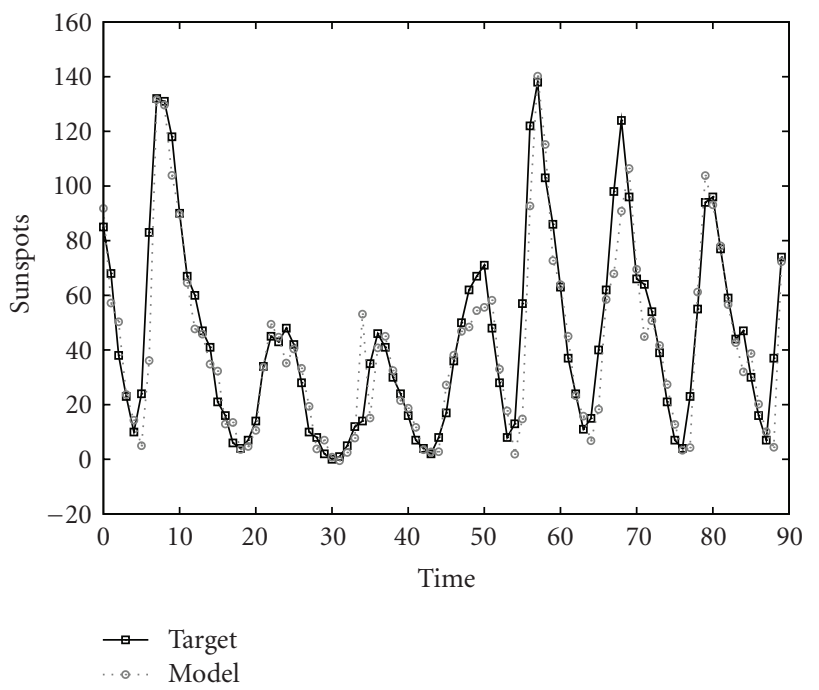

Figure 11: Symbolic regression Experiment 5: Target versus Model.

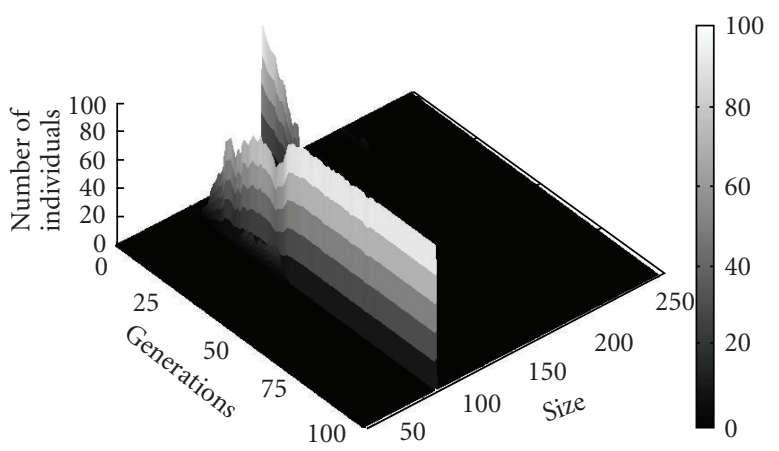

FIGURE 12: Symbolic regression Experiment 4: chromosome size in the population.

4.3. Parameter Optimization Results. Table 4 shows a summary of the results of parameter optimization experiments. Included in the summary are the maximum value found, the number of genes used by the best solution, the average gene length (static for canonical GEP), and the total genome size. The visualized results and performance of the experiments are shown by Figures 15, 16, 17, 18, 19, 20, 21, 22, 23 and 24.

\subsubsection{Discussion of Parameter Optimization Experiments.} The results of the parameter optimization experiments show that both our methodology and canonical GEP are effective at evolving either optimal or near-optimal solutions to the problems in the De Jong test suite. As seen in previous experiment series, our enhancements enabled the algorithm

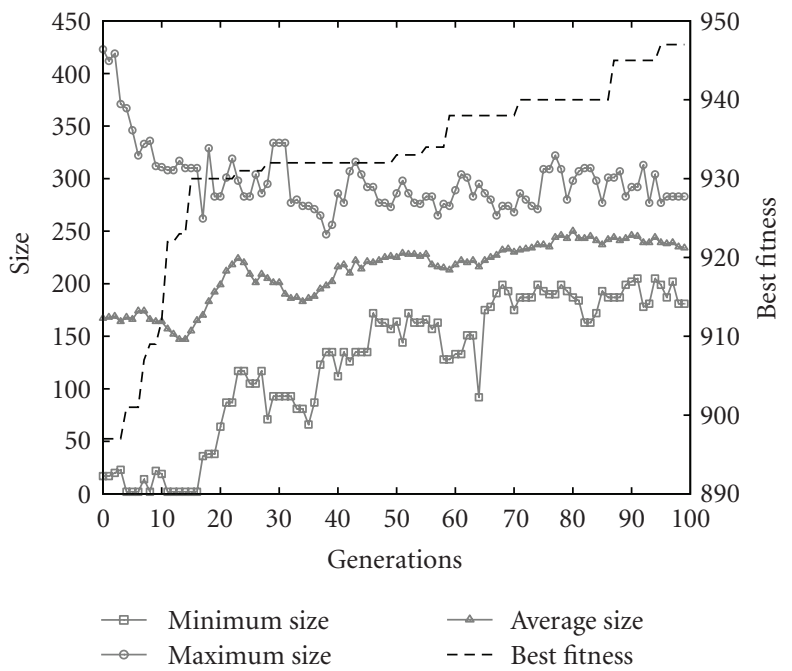

Figure 13: LiveDescribe experiment: chromosome sizes and best fitness.

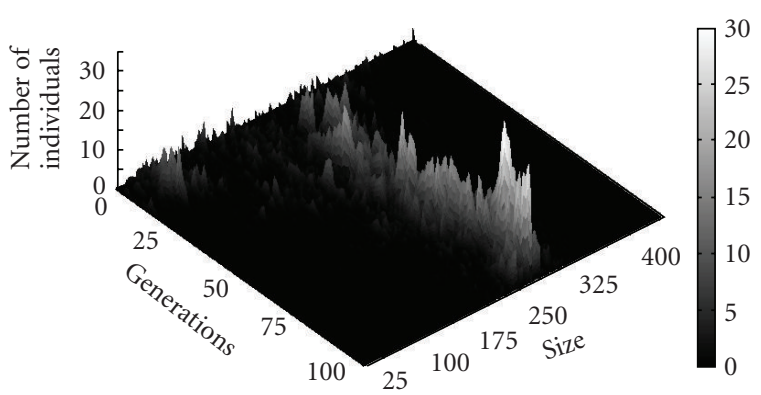

Figure 14: LiveDescribe experiment: chromosome size in the population.

to consistently evolve solutions which were significantly smaller than those evolved by canonical GEP.

The solutions evolved by our enhanced algorithm in Experiments 2 and 3 were remarkably smaller than those found by canonical GEP. Specifically, they were 5.4\% and $6.5 \%$ the size of those found by standard GEP.

Both methodologies had difficulty with the highdimension problem found in parameter optimization Experiment 4. However, our enhanced GEP algorithm evolved a slightly better result and had a representation size $54.6 \%$ the size of the one evolved by the standard algorithm. It is believed that the difficultly of this problem and the inability of the algorithm to locate the optimal parameter values contributed to the evolved size of the genome. Similarly, the numerical results of Experiment 1 were comparable, but the solutions evolved using the HIS 
TABLE 4: Summary of parameter optimization experimental results.

\begin{tabular}{lcccccc}
\hline $\begin{array}{l}\text { Exper. } \\
\text { Number }\end{array}$ & Total Len. & Ovg. Gene Len. & Maximum & Total Len. & \multicolumn{2}{c}{ Canonical GEP } \\
Gene Len.
\end{tabular}

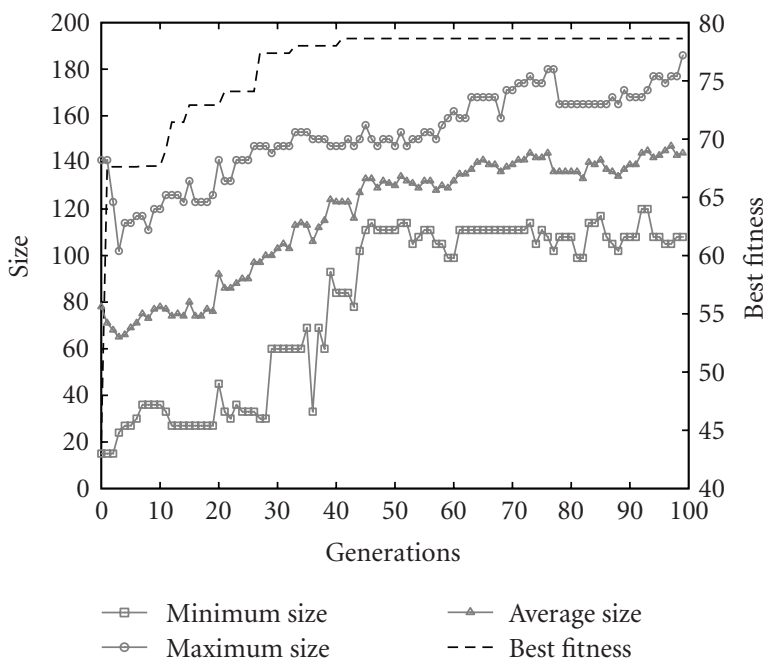

Figure 15: Parameter optimization Experiment 1: chromosome sizes and best fitness.

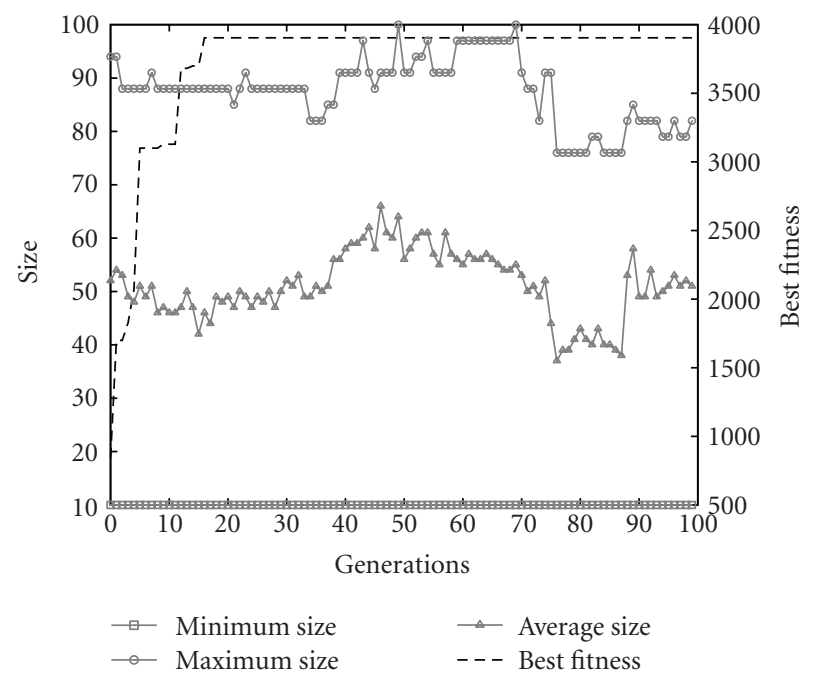

Figure 16: Parameter optimization Experiment 2: chromosome sizes and best fitness.

operator and our other enhancements were $45.5 \%$ the size of standard GEP's solutions.

The results of the final parameter optimization experiment were closer to what we had observed during the Symbolic Regression and Classification experiments, with our evolved solutions being approximately $23.4 \%$ the size

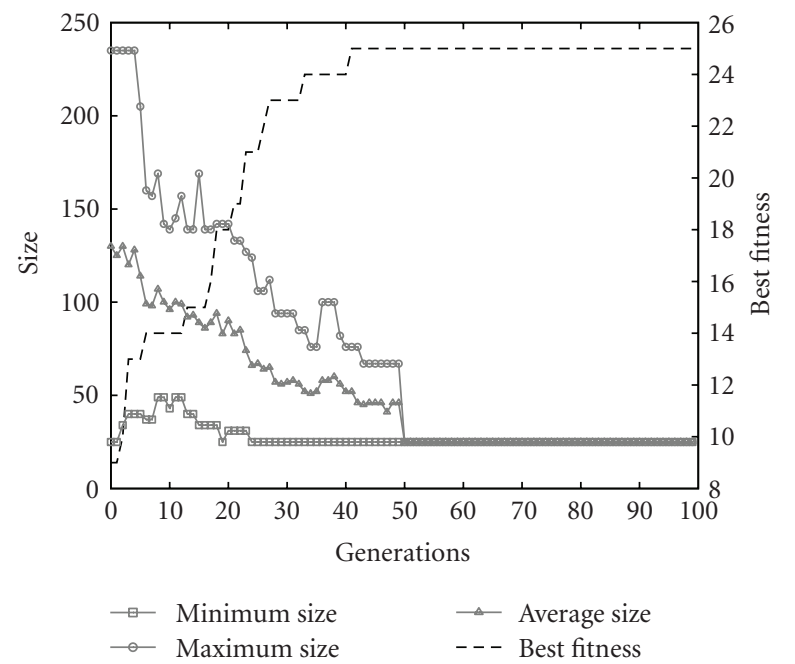

Figure 17: Parameter optimization Experiment 3: chromosome sizes and best fitness.

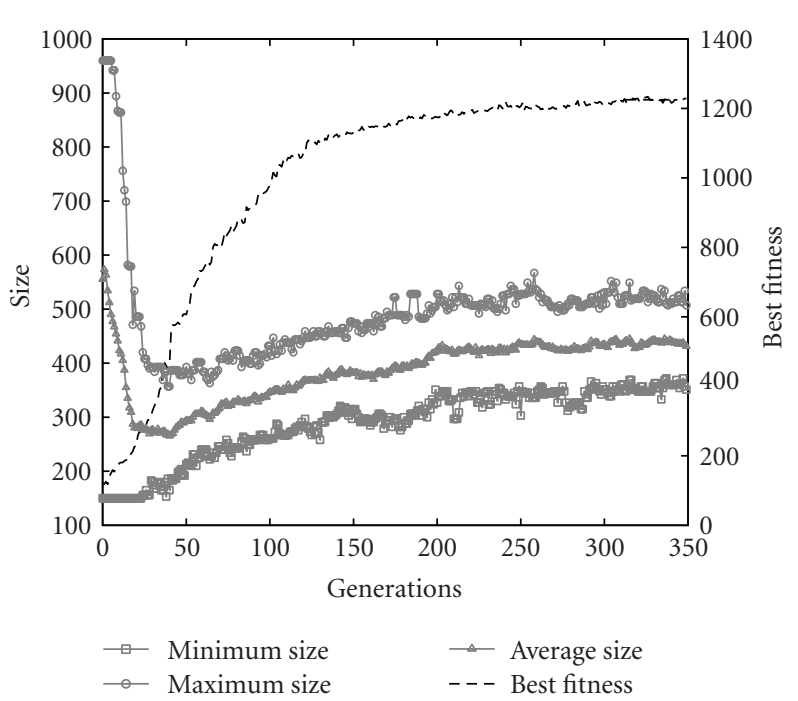

FIgURE 18: Parameter optimization Experiment 4: chromosome sizes and best fitness.

of those evolved by canonical GEP. In this case, both methodologies successfully found the maximum value of Shekel's foxholes.

All of the parameter optimization experiments have shown that our enhancements retained GEP's problem 


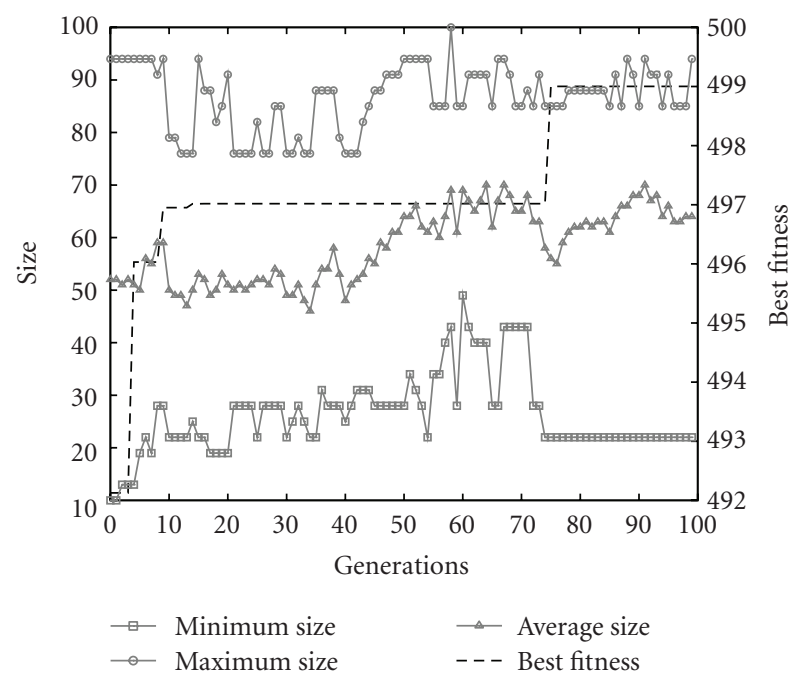

Figure 19: Parameter optimization Experiment 5: chromosome sizes and best fitness.

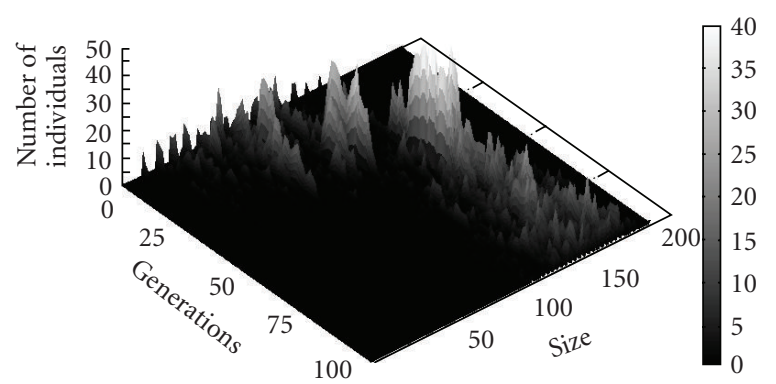

Figure 20: Parameter optimization Experiment 1: chromosome size in the population.

solving ability while allowing it to evolve smaller genomes. While the De Jong functions have been reported [48] to not be an effective test set, they have been repeatedly shown to provide a good metric of the effectiveness of algorithms for a broad range of optimization problems.

A possible limitation is that it is not currently possible to use the ACS mutation operator with our current experimental setup. Since we have not used Automatically Defined Functions (ADFs) [1], we must use a fixed number of genes, one per parameter requiring optimization. While we were still able to obtain good results, we can only speculate that using ADFs and allowing the number of "normal" genes to evolve (similarly to our symbolic regression and classification experiments) would enhance the solutions of more difficult parameter optimization problems.

4.4. General Discussions. Reviewing the results of our experiments, we see that our enhancements to the GEP algorithm consistently produced smaller solutions (sometimes significantly so) than canonical GEP. Since the representation size of a genome has a direct impact on the evaluation of the fitness cases, the reduction in representation size may improve the overall performance of the system, even after considering the additional computation requirements

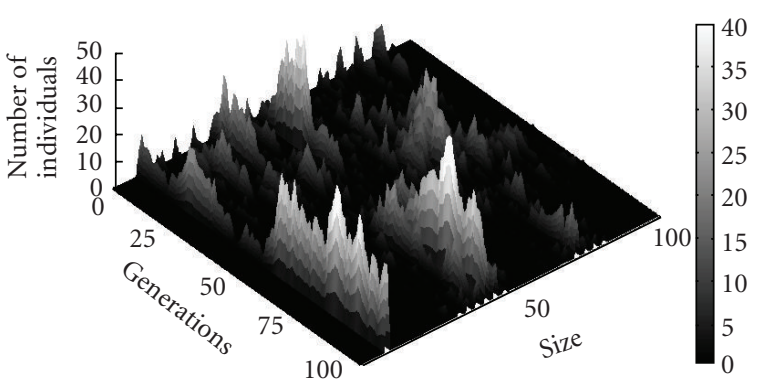

FIgure 21: Parameter optimization Experiment 2: chromosome size in the population.

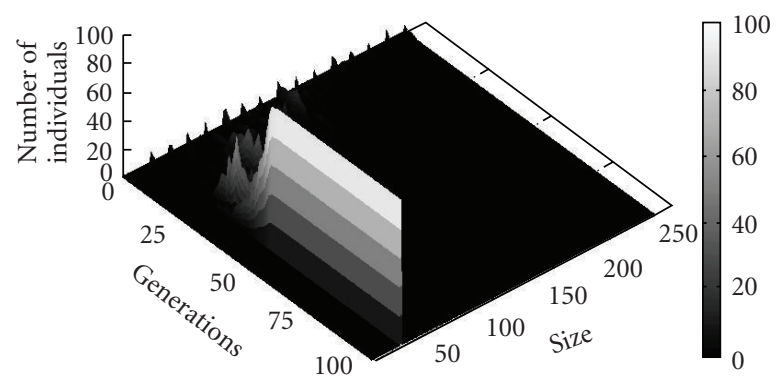

Figure 22: Parameter optimization Experiment 3: chromosome size in the population.

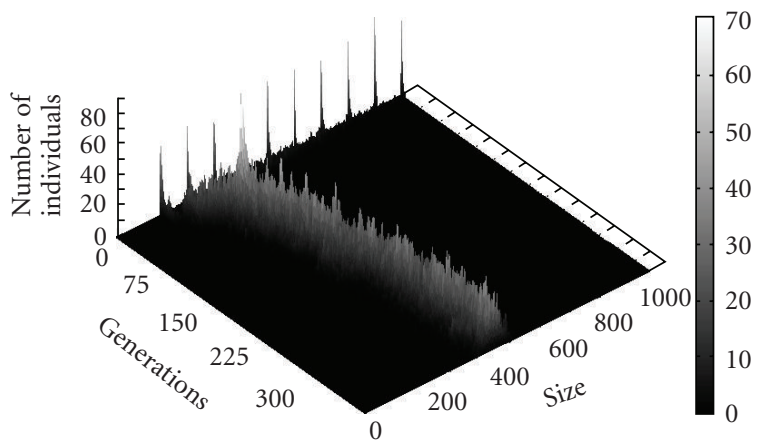

FIGURE 23: Parameter optimization Experiment 4: chromosome size in the population.

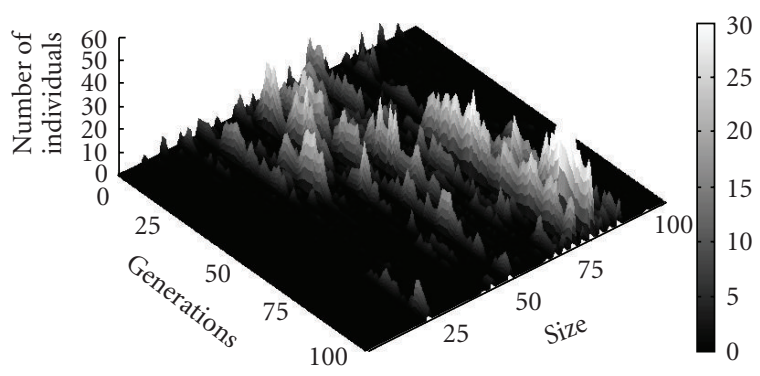

FIgure 24: Parameter optimization Experiment 5: chromosome size in the population. 
of our new operators. This was indirectly observed during the classification experiments while waiting for the two methodologies to complete their evolutionary runs. When our enhanced algorithm was running, it was noticeably faster than when the standard GEP algorithm was processing the same problem.

The control of the number of genes and the head size of each gene was an implicit part of our GEP run and, thus, we did not require separate clients for optimization. This reduced the overall computational resources required to evolve solutions.

For all of the parameter optimization experiments the ACS mutation operator was disabled and, thus, we were unable to evaluate its potential effectiveness for this class of problems. The operator was disabled because of the evaluation method used. Since our GEP implementation did not use ADFs, it required one gene per parameter to optimize. It is possible that, if we implemented automatically defined functions and used the ACS mutation operator to evolve the number of "normal" genes, we would see different results.

The decision to randomly initialize the genes that were inserted during the ACS mutation phase appears successful. However, it would be interesting to investigate the use of gene cloning, or other methods, in the future.

We observed that the insertion point in the ACS mutation operator for classification and symbolic regression problems was not important because we used a commutative linking function during testing. The insertion point, however, may have been significant because of the way the gene would mix within the population during recombination. Additionally, since the Gene Transposition operator was used, good genes could be reordered within the chromosome. Had we used a noncommutative linking function or homeotic (ADF) genes, the insertion location could have had a greater impact.

Based on the results of our experiments, our new operators were able to successfully self-adaptively tune the two critical parameters of the GEP algorithm, the head domain length, and the number of genes. While our new operators have additional computational costs associated with them, it is hoped that they are offset by the shorter time required to evaluate the fitness functions, because of the smaller representations it evolved.

Our new recombination operators have also been empirically shown to permit crossbreeding and speciation within a single GEP population. They have also been shown to be effective in a distributed environment. However, additional research into the effects of our operators on migration is required.

\section{Conclusion}

This work presented enhancements to the Gene Expression Programming algorithm that enabled flexible genome representations, endowed self-adaptive characteristics, assisted with maintaining diversity within a population and enhanced the parallelization of the algorithm. In particular, the enhancements addressed issues of evolvability, crossbreeding and speciation, parameter control, and parallelization in canonical GEP.

Through a series of experiments that used an assortment of problem classes, including symbolic regression, classification, and parameter optimization, we have shown that our proposed methodology produced better results and, generally, smaller genome representations than the canonical GEP algorithm and the PGEP-O system [3] (for symbolic regression).

Specifically, the contributions presented in this work were

(1) creation of a new transposition operator, the Head Insertion Sequence (HIS), which self-adaptively tunes the head domain length of a gene,

(2) development of a new mutation operator, the Adaptive Chromosome Size (ACS) mutation, which mutates the number of genes in an individual to tune the-gene count parameter,

(3) addition of new GEP recombination operators to permit structurally dissimilar individuals to interact. which removed the structural constraints imposed when transferring an individual from one population to another and permitted both crossbreeding and speciation.

Our enhancements to the GEP algorithm also simplified its use, by implicitly controlling the head domain length and number of genes throughout an evolutionary run. By removing the need to set these two critical GEP parameters prior to executing a run, the level of "expert knowledge" required to use GEP is reduced and allows EC novices to use the algorithm more effectively.

The simplification of the algorithm's configuration and the implicit parameter control of the two critical parameters are still subject to the concept of "No Free Lunch" [35]. The "No Free Lunch" theorem states that without a priori knowledge of a problem all potential solution methods are equal. While the values of the parameters evolved during a run may not be optimal for all problem types, they are frequently "good enough" and "No Free Lunch" is partially offset by the ease of using the new algorithm. This was seen during our experimental verification of the algorithm and when comparing our methodology to canonical GEP. To determine the GEP experimental baselines, several runs with different head domain length and number of gene parameter values were required, to obtain usable results. Comparatively, with our enhanced algorithm we only needed to start a run sequence and let the algorithm evolve the parameters.

While our enhancements to the GEP algorithm have proven to be successful, they are not without costs and limitations. Since we have added extra operators to enable our metaevolution of the parameters, we also have added additional computational overhead. In particular, the ACS mutation operator has significant overhead when it generates a new gene from random elements. The overhead associated with the new operators may be partially offset by the reduced size of the solution representations (as experienced during 
our trials), but further experimentation and analysis are required to confirm this.

Another side effect of our self-adaptive method is that we have increased the search space available to the algorithm. This is both a benefit and a liability, since the algorithm can traverse the entire space defined by any combination of head domain length and number of genes. This allows the algorithm to find novel solutions, but also increases the number of potential solutions dramatically, possibly increasing the search time and allowing the algorithm to get stuck in at a nonoptimal solution.

When developing the enhancements to the GEP algorithm, the possibility of introducing bloat, or the excessive creation of introns to protect a genome's functionality, was a major concern. By eliminating the fixed chromosome size (which was necessary to remedy the issues we saw with GEP), the potential for the genome representation and size to grow unchecked became a possibility; even with the parameter control inherent in the new operators. One conjecture for not observing bloat is that the HIS Transposition operator, which is responsible for controling the head size, restructures the genome by adding sections from one domain to another, instead of simply inserting or deleting material. This does not account for the effect of the ACS mutation operator, which mutates the number of genes in a chromosome. However, the selection pressure from the Tournament Selection with Elitism selection method may have provided resistance to unnecessary gene additions. It is possible that in more difficult problems (that require longer runs or larger datasets) we may begin to observe bloat and need to take steps to measure and constrain it.

Related to the previous topic of bloat and introns is the matter of genetic diversity within a population. Our current research did not include any specific mechanisms to measure the diversity of individuals within a population (either in a single population or distributed multipopulation setting), but the genome length statistics, recorded during the experiments, can be used as a simple metric. Using the surface plots of the chromosome lengths (found in Section 4), we can suppose that our methodology maintains a level of genetic diversity throughout a run. While the populations were initially very diverse and chaotic, as the runs progressed, the outliers were reduced and a narrower band of chromosome sizes (and thus diversity) was maintained.

Overall, our enhancements have been shown to be effective at addressing the issues of evolvability, crossbreeding and speciation, parameter control, and parallelization in the canonical GEP algorithm.

5.1. Future Work. Though our enhancements have been effective, there is still work that can be done to further our understanding of them, their relationship and application to Evolutionary Computation in general, and the workings of the GEP algorithm itself.

A detailed study of the effects of our enhancements on the levels of genetic diversity in a population would aid in understanding the mechanisms that make the operators effective. Additionally, applying the "Nonsynonymous to
Synonymous Substitution Ratio (Ka/Ks)" [49] to study the rate of evolution, in conjunction with a diversity study, could show where further improvements could be made in the GEP algorithm.

Applying our enhancements to Automatically Defined Functions (ADFs) in GEP could potentially provide interesting results and bears further investigation. This could be particularly useful for difficult or complex parameter optimization problems, since, when using GEP-PO, the number of genes must always equal the number of parameters being optimized. Using ADFs would allow the number of normal genes to be adaptively tuned using the ACS mutation operator.

Further research into the potential of unrestrained chromosome growth, or bloat, and selection pressure in our enhanced GEP algorithm would be interesting, as we did not observe significant bloat during our experiments. In evolutionary computation, any algorithm or representation that allows unrestrained growth and yet demonstrates resistance to bloat warrants further investigation.

The impact of our operators on migration and the exchange of genetic material in distributed setting requires further study. In particular, a thorough examination of our system when running in a distributed, multi-island settings with different connection topologies and migration strategies would be useful for determining the optimal configuration (if possible).

While the enhancements presented in this work enabled crossbreeding and the evolution of different species within a population, we did not specifically implement any niching methods. This could prove to be an interesting avenue of exploration in the future, as it could enhance the algorithm's performance with multimodal problems.

Finally, adapting our enhancements to neuroevolution, or the evolution of neural networks, using GEP (such as the GEP-nets algorithm [7]) has great potential. This is because our enhancements could permit size and structure changes to the evolved neural networks, allowing a more dynamic and complicated structure to be evolved.

\section{References}

[1] C. Ferreira, "Gene expression programming: a new adaptive algorithm for solving problems," Complex Systems, vol. 13, no. 2, pp. 87-129, 2001.

[2] K. A. De Jong, Analysis of the behavior of a class of genetic adaptive systems, Ph.D. dissertation, University of Michigan, 1975.

[3] H.-H. Park, A. Grings, M. V. dos Santos, and A. S. Soares, "Parallel hybrid evolutionary computation: automatic tuning of parameters for parallel gene expression programming," Applied Mathematics and Computation, vol. 201, no. 1-2, pp. 108-120, 2008.

[4] K. Zhang, S. Sun, and H. Si, "Prediction of retention times for a large set of pesticides based on improved gene expression programming," in Proceedings of the 10th Annual Conference on Genetic and Evolutionary Computation (GECCO '08), pp. 1725-1726, ACM, Atlanta, Ga, USA, July 2008.

[5] Z. Xie, X. Li, B. Di Eugenio, P. C. Nelson, W. Xiao, and T. M. Tirpak, "Using gene expression programming to 
construct sentence ranking functions for text summarization," in Proceedings of the 20th International Conference on Computational Linguistics (COLING '04), p. 1381, Association for Computational Linguistics, Morristown, NJ, USA, 2004.

[6] J. Venter and A. Hardy, "Generating plants with gene expression programming," in Proceedings of the ACM International Conference on Computer Graphics, Virtual Reality and Visualisation in Africa (AFRIGRAPH '07), pp. 159-167, ACM, 2007.

[7] C. Ferreira, Gene Expression Programming: Mathematical Modeling by an Artificial Intelligence, Springer, Berlin, Germany, 2nd edition, 2006.

[8] M. Ostaszewski, P. Bouvry, and F. Seredynski, "Multiobjective classification with moGEP: an application in the network traffic domain," in Proceedings of the 11th Annual Genetic and Evolutionary Computation Conference (GECCO '09), pp. 635642, ACM, 2009.

[9] J. Yin, L. Huo, L. Guo, and J. Hu, "Short-term load forecasting based on improved gene expression programming," in Proceedings of the 7th World Congress on Intelligent Control and Automation (WCICA '08), pp. 5647-5650, Chongqing, China, June 2008.

[10] J. Koza, Genetic Programming: On the Programming of Computers by Means of Natural Selection, The MIT Press, Cambridge, Mass, USA, 1992.

[11] D. E. Goldberg, Genetic Algorithms in Search, Optimization, and Machine Learning, Addison-Wesley, Reading, Mass, USA, 1989.

[12] J. Reisinger, K. O. Stanley, and R. Miikkulainen, "Towards an empirical measure of evolvability," in Proceedings of the Workshops on Genetic and Evolutionary Computation (GECCO '05), pp. 257-264, ACM, Washington, DC, USA, June 2005.

[13] K. O. Stanley, Efficient evolution of neural networks through complexification, Ph.D. dissertation, The University of Texas at Austin, Austin, Tex, USA, 2004.

[14] H. S. Lopes and W. R. Weinert, "Egipsys: an enhanced gene expression programming approach for symbolic regression problems," International Journal of Applied Mathematics and Computer Science, vol. 14, no. 3, pp. 375-384, 2004.

[15] J. Yue, T. Chang-Jie, Z. Hai-Chun, et al., "Adaptive gene expression programming algorithm based on cloud model," in Proceedings of the 1st International Conference on BioMedical Engineering and Informatics (BMEI '08), vol. 1, pp. 226-230, May 2008.

[16] C. Tang, L. Duan, J. Peng, H. Zhang, and Y. Zong, "The strategies to improve performance of function mining by gene expression programming: genetic modifying, overlapped gene, backtracking and adaptive mutation," in Proceedings of the 17th Data Engineering Workshop, 2006.

[17] O. M. Shir and T. Back, "Niching methods: speciation theory applied for multi-modal function optimization," in Algorithmic Bioprocesses, pp. 705-729, Springer, Berlin, Germany, 2009.

[18] J. H. Holland, Adaptation in Natural and Artificial Systems: An Introductory Analysis with Applications to Biology, Control and Artificial Intelligence, University of Michigan Press, Ann Arbor, Mich, USA, 1975.

[19] E. Bautu, A. Bautu, and H. Luchian, "AdaGEP-an adaptive gene expression programming algorithm," in Proceedings of the 9th International Symposium on Symbolic and Numeric Algorithms for Scientific Computing (SYNASC '07), pp. 403406, 2007.

[20] M. Gorges-Schleuter, "Explicit parallelism of genetic algorithms through population structures," Parallel Problem Solving from Nature, pp. 150-159, 1991.
[21] J. I. Hidalgo, J. Lanchares, F. Fernández de Vega, and D. Lombraña, "Is the island model fault tolerant?" in Proceedings of the 9th Annual Genetic and Evolutionary Computation Conference (GECCO '07), pp. 2737-2744, ACM, London, UK, July 2007.

[22] E. Cantú-Paz and D. E. Goldberg, "Efficient parallel genetic algorithms: theory and practice," Computer Methods in Applied Mechanics and Engineering, vol. 186, no. 2-4, pp. 221$238,2000$.

[23] J. Berntsson and M. Tang, "Dynamic optimization of migration topology in internet-based distributed genetic algorithms," in Proceedings of the Genetic and Evolutionary Computation Conference (GECCO '05), pp. 1579-1580, ACM, Washington, DC, USA, June 2005.

[24] E. Cantu-Paz, Efficient and Accurate Parallel Genetic Algorithms, Kluwer Academic Publishers, Dordrecht, The Netherlands, 2000.

[25] E. Alba and J. M. Troya, "Analyzing synchronous and asynchronous parallel distributed genetic algorithms," Future Generation Computer Systems, vol. 17, no. 4, pp. 451-465, 2001.

[26] Z. Skolicki and K. De Jong, "The influence of migration sizes and intervals on island models," in Proceedings of the Genetic and Evolutionary Computation Conference (GECCO '05), pp. 1295-1302, ACM, Washington, DC, USA, June 2005.

[27] Z. Skolicki and K. De Jong, "The importance of a two-level perspective for island model design," in Proceedings of IEEE Congress on Evolutionary Computation (CEC '07), pp. 46234630, 2007.

[28] S.-K. Oh, C. T. Kim, and J.-J. Lee, "Balancing the selection pressures and migration schemes in parallel genetic algorithms for planning multiple paths," in Proceedings of IEEE International Conference on Robotics and Automation, vol. 4, pp. 3314-3319, 2001.

[29] S.-C. Lin, W. F. Punch III, and E. D. Goodman, "Coarse-grain parallel genetic algorithms: categorization and new approach," in Proceeedings of the 6th IEEE Symposium on Parallel and Distributed Processing, pp. 28-37, Dallas, Tex, USA, October 1994.

[30] E. Alba and J. M. Troya, "Influence of the migration policy in parallel distributed GAs with structured and panmictic populations," Applied Intelligence, vol. 12, no. 3, pp. 163-181, 2000.

[31] Y. Lin, H. Peng, and J. Wei, "A niching gene expression programming algorithm based on parallel model," in Proceedings of the 7th International Symposium on Advanced Parallel Processing Technologies (APPT'07), vol. 4847 of Lecture Notes in Computer Science, pp. 261-270, Guangzhou, China, November 2007.

[32] J. Wu, C. Tang, T. Li, S. Qiao, Y. Jiang, and S. Ye, "Parallel multi-objective gene expression programming based on area penalty," in Proceedings of the International Conference on Computer Science and Information Technology (ICCSIT '08), pp. 264-268, Singapore, August-September 2008.

[33] Q. Liu, T. Li, C. Tang, Q. Liu, C. Li, and S. Qiao, "Multipopulation parallel genetic algorithm for economic statistical information mining based on gene expression programming," in Proceedings of the 3rd International Conference on Natural Computation (ICNC '07), vol. 3, pp. 461-465, August 2007.

[34] X. Du, L. Ding, and J. Jia, "Asynchronous distributed parallel gene expression programming based on estimation of distribution algorithm," in Proceedings of the 4th International Conference on Natural Computation (ICNC '08), vol. 1, pp. 433-437, Jinan, China, October 2008. 
[35] D. H. Wolpert and W. G. Macready, "No free lunch theorems for optimization," IEEE Transactions on Evolutionary Computation, vol. 1, no. 1, pp. 67-82, 1997.

[36] Y. C. Ho and D. L. Pepyne, "Simple explanation of the no-freelunch theorem and its implications," Journal of Optimization Theory and Applications, vol. 115, no. 3, pp. 549-570, 2002.

[37] A. E. Eiben, Z. Michalewicz, M. Schoenauer, and J. E. Smith, "Parameter control in evolutionary algorithms," Studies in Computational Intelligence, vol. 54, pp. 19-46, 2007.

[38] T. Back, "Self-adaptation in genetic algorithms," in Proceedings of the 1st European Conference on Artificial Life, pp. 263-271, MIT Press, 1992.

[39] M. Hai-Jun, LI De-Yi, and S. Xue-Mei, "Membership clouds and membership cloud generators," Journal of Computer Research and Development, pp. 15-20, 1995.

[40] C. Ferreira, "Q\&a from personal correspondence," http://www.gene-expression-programming.com/Q\&A03.asp.

[41] J. Branke, A. Kamper, and H. Schmeck, "Distribution of evolutionary algorithms in heterogeneous networks," in Proceedings of the Genetic and Evolutionary Computation Conference (GECCO '04), vol. 3102 of Lecture Notes in Computer Science, pp. 923-934, Seattle, Wash, USA, June 2004.

[42] R. I. McKay, X. H. Nguyen, J. R. Cheney, M. Kim, N. Mori, and T. H. Hoang, "Estimating the distribution and propagation of genetic programming building blocks through tree compression," in Proceedings of the 11th Annual Genetic and Evolutionary Computation Conference (GECCO '09), pp. 1011-1018, ACM, 2009.

[43] T. W. Anderson, The Statistical Analysis of Time Series, John Wiley \& Sons, New York, NY, USA, 1971.

[44] T. C. for Learning Technology (CLT) at Ryerson University, "Livedescribe video description software," September 2009, http://www.livedescribe.com/.

[45] H. Rosenbrock, "An automatic method for finding the greatest or least value of a function," The Computer Journal, vol. 3, pp. 175-184, 1960.

[46] J. Shekel, "Test functions for multimodal search techniques," in Proceedings of the 5th Annual Princeton Conference on Information Science and Systems, 1971.

[47] J. Alami, A. E. Imrani, and A. Bouroumi, "A multipopulation cultural algorithm using fuzzy clustering," Applied Soft Computing Journal, vol. 7, no. 2, pp. 506-519, 2007.

[48] L. D. Whitley, K. E. Mathias, S. B. Rana, and J. Dzubera, "Building better test functions," in Proceedings of the 6th International Conference on Genetic Algorithms, pp. 239-247, 1995.

[49] T. Hu and W. Banzhaf, "Nonsynonymous to synonymous substitution ratio ka/ks: measurement for rate of evolution in evolutionary computation," in Proceedings of the 10th International Conference on Parallel Problem Solving from Nature, pp. 448-457, Springer, 2008. 

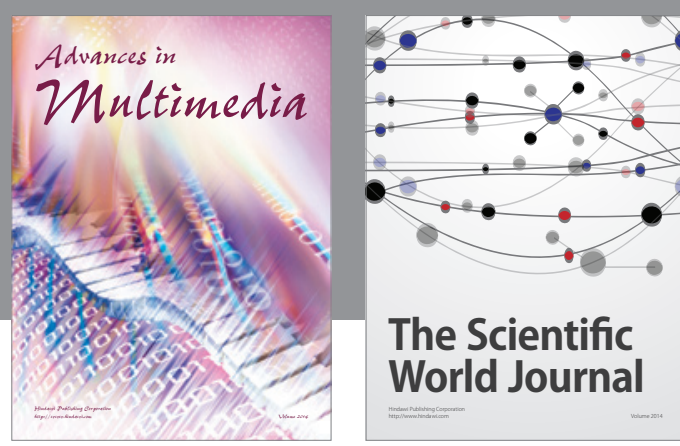

The Scientific World Journal
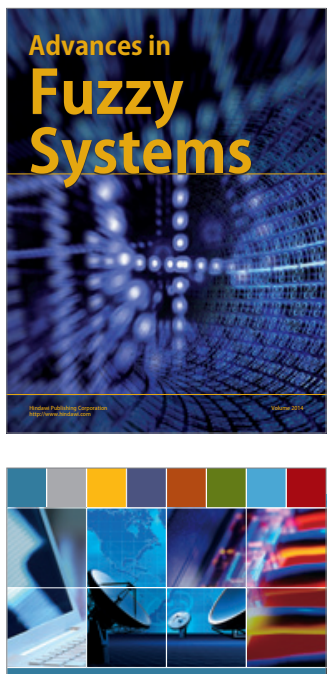

Computer Networks and Communications
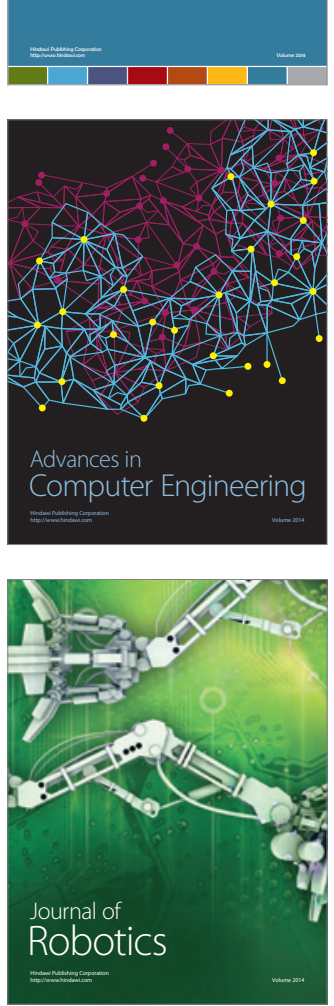
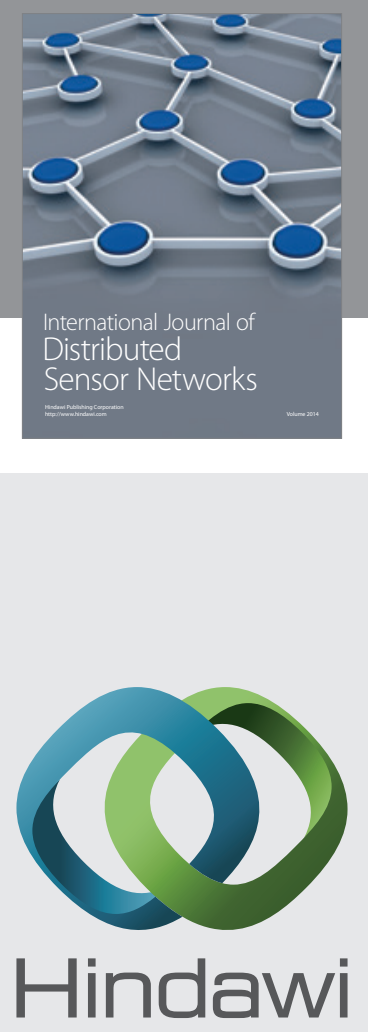

Submit your manuscripts at

http://www.hindawi.com
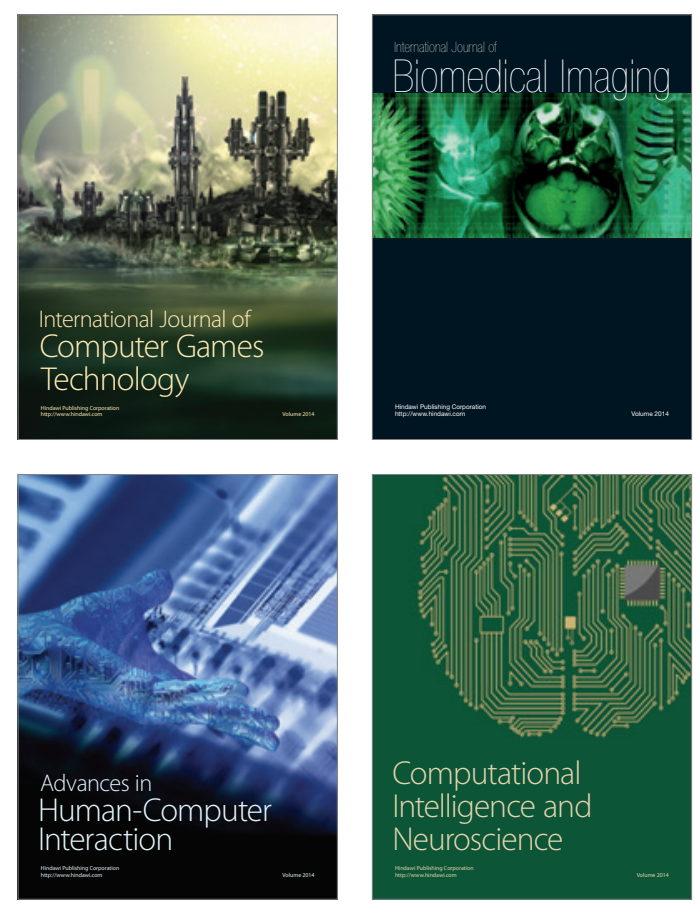
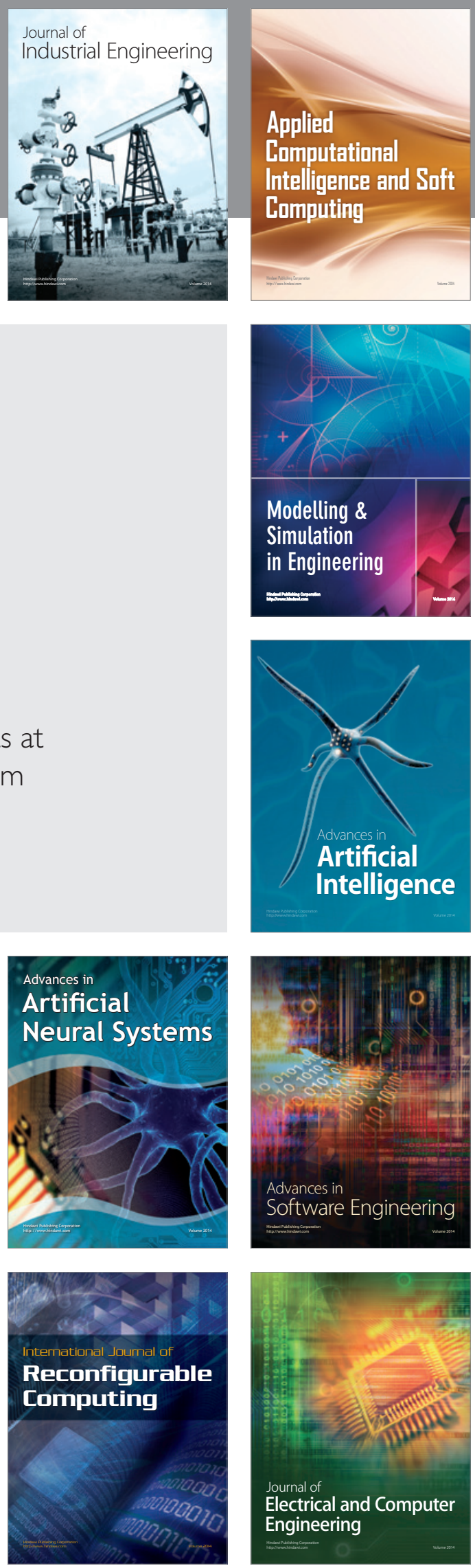